\begin{tabular}{|r|l|}
\hline \multicolumn{2}{|c|}{ Statistica Sinica Preprint No: SS-2020-0214 } \\
\hline Title & $\begin{array}{l}\text { Copula-Based Functional Bayes Classification With } \\
\text { Principal Components and Partial Least Squares }\end{array}$ \\
\hline Manuscript ID & SS-2020-0214 \\
\hline URL & http://www.stat.sinica.edu.tw/statistica/ \\
\hline DOI & $10.5705 /$ ss.202020.0214 \\
\hline Complete List of Authors & $\begin{array}{l}\text { Wentian Huang and } \\
\text { David Ruppert }\end{array}$ \\
\hline Corresponding Author & Wentian Huang \\
\hline E-mail & wh365@cornell.edu \\
\hline Notice: Accepted version subject to English editing.
\end{tabular}




\title{
COPULA-BASED FUNCTIONAL BAYES CLASSIFICATION WITH PRINCIPAL COMPONENTS AND PARTIAL LEAST SQUARES
}

\author{
Wentian Huang, David Ruppert
}

Cornell University

\begin{abstract}
We present a new functional Bayes classifier that uses principal component (PC) or partial least squares (PLS) scores from the common, that is, pooled, covariance function, that is, the covariance function marginalized over groups. When the groups have different covariance functions, the PC or PLS scores need not be independent or even uncorrelated. We use copulas to model the dependence. Our method is semiparametric; the marginal densities are estimated nonparametrically by kernel smoothing and the copula is modeled parametrically. We focus on Gaussian and $t$-copulas, but other copulas could be used. The strong performance of our methodology is demonstrated through simulation, real data examples, and asymptotic properties.
\end{abstract}

Key words and phrases: Asymptotic theory, Bayes classifier, functional data, perfect classification, rank correlation, semiparametric model 


\section{Introduction}

Functional classification, where the features are continuous functions on a compact interval, has received increasing interest in recent years, e.g., in chemometrics, medicine, economics and environmental science. James and Hastie (2001 [17]) extended linear discriminant analysis (LDA) to functional data (FLDA), including the case where the curves are partially observed. James (2002) [16] proposed a functional version of the generalized linear model (FGLM) including functional logistic regression. Thereafter, FGLM was further researched by works like Müller and Stadtmüller (2005) [28], Li et al. (2010) [22], Zhu et al. (2010) [36], McLean et al. (2014) [26], Shang and Cheng (2015) [33], among many others. Aside from FGLM, other classifiers are also studied: Rossi and Villa (2006 [31]) applied support vector machines (SVM) to classify infinite-dimensional data; Cuevas et al. (2007 [6]) explored classification of functional data based on data depth; Li and Yu (2008 [21]) suggested a functional segmented discriminant analysis combining LDA and SVM; Cholaquidis et al. (2016 [3]) proposed a nonlinear aggregation classifier.

However, certain issues remain. Current methods, e.g., FLDA, SVM, and the functional centroid classifier (Delaigle and Hall, 2012a [9]), distinguish groups by differences between their functional means. They achieve satisfactory results when the location difference is the dominant feature distinguishing classes, but functional data provide more information than just group means. For example, Fig. 1 from the example in Section 4.1 compares mean and standard deviation functions of raw and smoothed fractional anisotropy (FA) measured along the corpus callosum (cca) of 141 subjects, 99 with multiple sclerosis (MS) and 42 without. The disparity between the group standard deviations in panel (c) provides additional information that can identify MS patients. As shown in Section 4.1, the LDA and 
centroid classifiers fail to capture this information and have higher misclassification rates than the classifiers we propose.
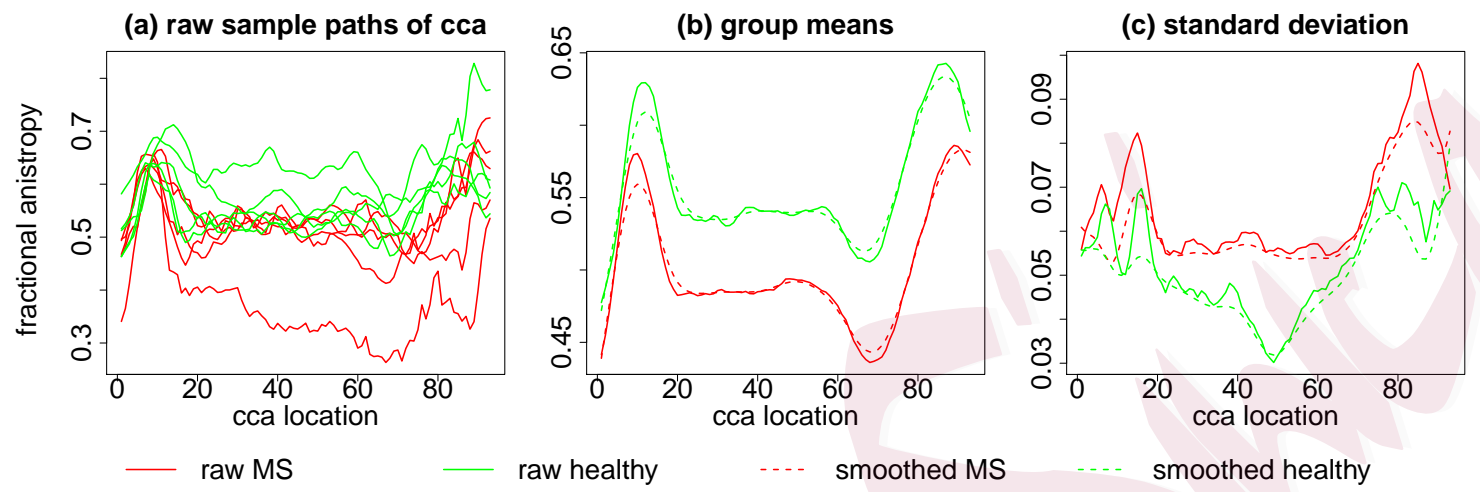

Figure 1: Panel (a) shows profiles of fractional anisotropy (FA), five each of cases and controls, while panels (b) and (c) show group means and standard deviations. MS cases are red, and solid versus dashed lines distinguish raw and smoothed data. Compared to controls, the MS group has both a lower mean and a higher standard deviation.

Both parametric and nonparametric methods have their own drawbacks in classifying functional data. Parametric models such as linear and quadratic discriminant analysis are popular in functional classification, especially since nonparametric methods are likely to encounter the curse of dimensionality. However, parametric methods can cast rigid assumptions on the class boundaries ( $\mathrm{Li}$ and $\mathrm{Yu}, 2008$ [21]). Our interest is in methods that avoid stringent assumptions on the data. Dai et al. (2017 [7]) proposed a nonparametric Bayes classifier, assuming that the subgroups share the same sets of eigenfunctions, and that the scores projected on them are independent. With these assumptions and the definition of the density of random functions proposed by Delaigle and Hall (2010 [8]), joint densities of truncated functional data can be estimated by univariate kernel density estimation (KDE). The Bayes rules estimated this way avoid the curse of dimensionality, but require that the groups have equal sets of eigenfunctions and independent scores.

We propose new semiparametric Bayes classifiers. We project the functions onto the 
eigenfunctions of the pooled covariance function, that is, the covariance function marginalized over group. These eigenfunctions can be estimated by functional principal components analysis (fPCA) applied to the combined groups. The projections will not be independent or even uncorrelated, unless these common eigenfunctions are also the eigenfunctions of the group-specific covariance functions, an assumption not likely to hold in many situations. For instance, in Section 4 we discuss two real data examples, and include the comparison of their group eigenfunctions in the supplementary materials (Fig. S4 and Fig. S8). Both cases appear to violate the equal eigenfunction assumption. We estimate the marginal density of the projected scores by univariate KDE as in Dai et al. (2017 [7]) and model the association between scores using a parametric copula. Our semiparametric methodology avoids the restricted range of applications imposed by the assumption of equal group-specific eigenfunctions. It also avoids the curse of dimensionality that multivariate nonparametric density estimation would entail.

Besides the principal components (PC) basis, we also consider a partial least squares (PLS) projection basis. Partial least squares has attracted recent attention due to its effectiveness in prediction and classification problems with high-dimensional and functional data. Preda et al. (2007 [29]) discussed functional LDA combined with PLS. Delaigle and Hall (2012a [9]) mentioned the potential advantage of PLS scores in their functional centroid classifier, when the difference between the group means does not lie primarily in the space spanned by the first few eigenfunctions. We find that PLS scores can be more efficient than PC scores in capturing group mean differences.

This article presents main advances over previous works by two aspects: in numerical results, the new method shows improved prediction accuracy and strength in dimension 
reduction, and extends the functional Bayes classification to multiclass classification; in the theoretical analysis, several new conditions are added for the functional data to achieve asymptotic optimality. These conditions are required because of the unequal group-specific eigenfunctions. Moreover, we propose asymptotic sparsity assumptions on the inverse of the copula correlations in our new method, following the design of Yuan (2010 [35]) and Liu et al. (2012 [23]) for high dimensional data. We also build a new theorem which utilizes the special copula structure to achieve asymptotic perfect classification.

In Section 2, we introduce our model and the copula-based functional Bayes classifiers. Section 3 contains a comprehensive simulation study comparing our methods with existing classifiers on both binary and multiclass problems. Section 4 uses two real data examples to show the strength of our classifiers in accuracy and dimension reduction with respect to data size. In Section 5, we discuss the asymptotic properties of our classifiers. We also establish conditions for our classifier to achieve perfect classification on data generated by both Gaussian and non-Gaussian processes. Finally, in Section 6 we discuss future work, especially about extending the classification to the case where there are multiple functional predictors. Additional results and detailed proofs are in the Supplementary Materials.

\section{Model Setup \& Functional Bayes Classifiers with Copulas}

\subsection{Methodology}

Suppose $\left(X_{i . .}, Y_{i}\right), i=1, \ldots, n$ are i.i.d. from the joint distribution of $(X, Y)$, where $X$ is a square integrable function over some compact interval $\mathcal{T}$, i.e., $X \in \mathcal{L}^{2}(\mathcal{T}) . \quad Y=0,1$ is an indicator of groups $\Pi_{0}$ and $\Pi_{1}$, and $\pi_{k}=P(Y=k)$. Also, $X_{i \cdot k}, i=1, \ldots, n_{k}$ and $k=0,1$, denotes the $i$-th sample curve of $X_{. . k}=(X \mid Y=k)$, and $n=\sum_{k=0,1} n_{k}$. Our goal is to classify a new observation, $x$. 
Note that throughout the article, we order the index of $X$ by observation counts $(i)$, joint basis $(j)$, and group labels $(k)$ : for curves, $X_{i \text {.. }}$ denotes the $i$-th observation of the random function $X, X_{. . k}$ as the random function $X \mid Y=k$, and therefore $X_{i \cdot k}$ as the $i$-th sample curve of $X_{. . k}$; additionally, $X_{. j .}$ and $X_{. j k}$ are random variables from projecting $X$ and $X_{. . k}$ respectively on $j$-th joint basis function $\psi_{j}$, with $X_{i j k}$ the $i$-th observation of $X_{\cdot j k}$.

Dai et.al. (2017 [7]) extended Bayes classification from multivariate to functional data: a new curve $x$ is classified into $\Pi_{1}$ if

$$
Q(x)=\frac{P(Y=1 \mid X=x)}{P(Y=0 \mid X=x)}=\frac{\bar{f}_{1}(x) \pi_{1}}{\bar{f}_{0}(x) \pi_{0}} \approx \frac{f_{1}\left(x_{1}, \ldots, x_{J}\right) \pi_{1}}{f_{0}\left(x_{1}, \ldots, x_{J}\right) \pi_{0}}>1,
$$

where $\bar{f}_{k}$ is the density of $X_{. . k}$ and $f_{k}$ is the joint density of scores $X_{. j k}$ on basis $\psi_{j}, 1 \leq j \leq J$.

A key feature of Bayes classification on functional data is that the classifiers vary by the choice of basis functions $\psi_{j}$ as well as the estimation of $f_{0}, f_{1}$. Dai et al. (2017 [7]) built the original functional Bayes classifier, which we will call BC (Bayes classifier), upon two important assumptions. First, the sets of the first $J$ eigenfunctions, $\left\{\phi_{1}, \ldots, \phi_{J}\right\}$, of the covariance operators $G_{1}$ and $G_{0}$ of the two groups are equal. Here $G_{k}\left(\phi_{j}\right)(t)=\int_{\mathcal{T}} G_{k}(s, t) \phi_{j}(s) d s=$ $\lambda_{j k} \phi_{j}(t), G_{k}(s, t)=\operatorname{cov}\left\{X_{. . k}(s), X_{. . k}(t)\right\}=\sum_{j=1}^{\infty} \lambda_{j k} \phi_{j}(s) \phi_{j}(t)$, and $\lambda_{j k}$ is the $j$-th eigenvalue in group $k$. Second, letting $\psi_{j}=\phi_{j}$ for $1 \leq j \leq J$, the $J$ projected scores $X_{\cdot j k}=\left\langle X_{. . k}, \phi_{j}\right\rangle$ are independent. Then with $f_{j k}$ as the marginal density of $X_{\cdot j k}$, the $\log$ ratio of $Q(x)$ in Eq.(2.1) becomes

$$
\log Q(x) \approx \log Q_{J}(x)=\log \left(\frac{\pi_{1}}{\pi_{0}}\right)+\sum_{j=1}^{J} \log \left\{\frac{f_{j 1}\left(x_{j}\right)}{f_{j 0}\left(x_{j}\right)}\right\}
$$

A classifier that uses Eq.(2.2) avoids the curse of dimensionality and only needs to es- 
timate the marginal densities, $f_{j k}$. However, as later simulations and examples show, its performance can be degraded if the two assumptions mentioned above are not met. We propose new semiparametric Bayes classifiers based on copulas, that do not require these two assumptions and yet are free from the curse of dimensionality. Theoretical work in Section 5 proves that these classifiers maintain the advantages of $\mathrm{BC}$ over a wider range of data distributions, and are capable of perfect classification when $n \rightarrow \infty$ and $J \rightarrow \infty$.

\subsection{Copula-Based Bayes Classifier with Principal Components}

Allowing for possibly unequal group eigenfunctions, the covariance function of group $k$ is

$$
G_{k}(s, t)=\operatorname{cov}\left(X_{. . k}(s), X_{. . k}(t)\right)=\sum_{j=1}^{\infty} \lambda_{j k} \phi_{j k}(s) \phi_{j k}(t), k=0,1
$$

with $\phi_{1 k}, \ldots, \phi_{J k}$ as eigenfunctions. For simplicity, we assume the group means are $E(X \mid Y=$ $0)=0$ and $E(X \mid Y=1)=\mu_{d}$. The joint covariance operator $G$ then has the kernel $G(s, t)=\pi_{1} G_{1}(s, t)+\pi_{0} G_{0}(s, t)+\pi_{1} \pi_{0} \mu_{d}(s) \mu_{d}(t)$.

As later examples suggest, the unequal group eigenfunction case is common. To accommodate this case, we can project data from both groups onto the same basis functions. Therefore, we use the eigenfunctions $\phi_{1}, \ldots, \phi_{J}$ of $G$ as the basis $\psi_{1}, \ldots, \psi_{J}$.

The joint density $f_{k}, k=0,1$ in Eq.(2.1) allows for potential score correlation and tail dependency, which we use copulas to model. A copula is a multivariate CDF whose univariate marginal distributions are all uniform, and it characterizes only the dependency between the components. See, for example, Ruppert and Matteson, 2015 [32]. Here we extend its use to the truncated scores of functional data.

Let $x_{j}=\left\langle x, \phi_{j}\right\rangle=\int_{\mathcal{T}} x(t) \phi_{j}(t) d t$ be the $j$ th projected score of $x$. The copula function 
$C_{k}$ describes the distribution of first $J$ scores in $\Pi_{k}$ by

$$
\begin{aligned}
F_{k}\left(x_{1}, \ldots, x_{J}\right) & =C_{k}\left\{F_{1 k}\left(x_{1}\right), \ldots, F_{J k}\left(x_{J}\right)\right\} \\
f_{k}\left(x_{1}, \ldots, x_{J}\right) & =c_{k}\left\{F_{1 k}\left(x_{1}\right), \ldots, F_{J k}\left(x_{J}\right)\right\} f_{1 k}\left(x_{1}\right) \cdots f_{J k}\left(x_{J}\right) .
\end{aligned}
$$

$F_{k}$ in Eq.(2.3) is the joint CDF of $X_{\cdot 1 k}, \ldots, X_{. J k}$, and $C_{k}$ is the CDF of the uniformly distributed variables $F_{1 k}\left(X_{\cdot 1 k}\right), \ldots, F_{J k}\left(X_{. J k}\right)$, where $F_{j k}$ is the univariate CDF of $X_{\cdot j k}$. In Eq.(2.4), the joint density $f_{k}$ is decomposed into score marginal densities $f_{j k}$ as well as the copula density $c_{k}$ for dependency between the projected scores. Our revised classifier is $\mathbb{1}\left\{\log Q_{J}^{*}(x)>0\right\}$, i.e. the new curve $x$ belongs to $\Pi_{1}$ if

$$
\log Q_{J}^{*}(x)=\log \left(\frac{\pi_{1}}{\pi_{0}}\right)+\sum_{j=1}^{J} \log \left\{\frac{f_{j 1}\left(x_{j}\right)}{f_{j 0}\left(x_{j}\right)}\right\}+\log \left\{\frac{c_{1}\left\{F_{11}\left(x_{1}\right), \ldots, F_{J 1}\left(x_{J}\right)\right\}}{c_{0}\left\{F_{10}\left(x_{1}\right), \ldots, F_{J 0}\left(x_{J}\right)\right\}}\right\}>0
$$

We also consider situations when $Y$ has more than two classes. A more general procedure for multiclass classification is described in the Supplementary Materials Section S2.

\subsection{Choice of Copula and Correlation Estimator}

There have been a number of approaches to copula estimation: Genest et al. (1995 [11]) studied asymptotic properties of semiparametric estimation in copula models; Chen and Fan (2006 [2]) discussed semiparametric copula estimation to characterize the temporal dependence in time series data; Kauermann et al. (2013 [18]) estimated copula density nonparametrically using penalized splines; Gijbels et al. (2012 [12]) applied multivariate kernel density estimation to copulas.

To address the high dimensionality of functional data, we model the copula densities $c_{1}$ 
and $c_{0}$ parametrically and use kernel estimation for the univariate densities $f_{1 k}, \ldots, f_{J k}, k=$ 0, 1. We study the properties of Bayes classification with both Gaussian and t-copulas, denote by BCG and BCt respectively. When $c_{k}$ is modeled by a Gaussian copula in Eq. $(2.4), c_{k}(\cdot)=$ $c_{G, k}\left(\cdot \mid \boldsymbol{\Omega}_{G, k}\right)$, where $c_{G, k}$ is the Gaussian copula density with $J \times J$ correlation matrix $\boldsymbol{\Omega}_{G, k}$. When there is tail dependency between the scores, a t-copula is used: $c_{k}(\cdot)=c_{t, k}\left(\cdot \mid \Omega_{t, k}, \nu_{k}\right)$, with $c_{t, k}$ the t-copula density, $\Omega_{t, k}$ the correlation matrix and $\nu_{k}$ the tail index.

There are several ways to estimate the correlation matrices $\Omega_{G, k}$ or $\Omega_{t, k}$. We use rank correlations, specifically, Kendall's $\tau$. Kendall's $\tau$ between the projected scores of $X_{. . k}$ on the $j$-th and $j^{\prime}$-th basis is $\rho_{\tau}\left(X_{\cdot j k}, X_{\cdot j^{\prime} k}\right)=E\left[\operatorname{sign}\left\{\left(X_{\cdot j k}^{(1)}-X_{\cdot j k}^{(2)}\right)\left(X_{\cdot j^{\prime} k}^{(1)}-X_{\cdot j^{\prime} k}^{(2)}\right)\right\}\right], \operatorname{sign}(x)=$ $\mathbb{1}\{x>0\}-\mathbb{1}\{x<0\}$, and $X_{. . k}^{(1)}, X_{. . k}^{(2)}$ as i.i.d. samples of $X_{. . k}$. Robustness of rank correlation, as well as its optimal asymptotic error rate, is studied by Liu et al. (2012 [23]).

A relationship exists between the $\left(j, j^{\prime}\right)$-th entry of the copula correlation $\Omega_{k}$ and Kendall's $\tau: \quad \Omega_{k}^{j j^{\prime}}=\sin \left(\frac{\pi}{2} \rho_{\tau}\left(X_{\cdot j k}, X_{\cdot j^{\prime} k}\right)\right)$ for both Gaussian and $t$-copulas (Kendall, 1948 [19]; Kruskal, 1958 [20]; Ruppert and Matteson, 2015 [32]). Then, $\boldsymbol{\Omega}_{k}^{j j^{\prime}}$ is estimated by Kendall's $\tau$ as $\hat{\Omega}_{k}^{j j^{\prime}}=\sin \left(\frac{\pi}{2} \hat{\rho}_{\tau, k}^{j j^{\prime}}\right)$, where

$$
\hat{\rho}_{\tau, k}^{j j^{\prime}}=\frac{2}{n_{k}\left(n_{k}-1\right)} \sum_{1 \leq i \leq i^{\prime} \leq n_{k}} \operatorname{sign}\left\{\left\langle X_{i \cdot k}-X_{i^{\prime} \cdot k}, \hat{\phi}_{j}\right\rangle\left\langle X_{i \cdot k}-X_{i^{\prime} \cdot k}, \hat{\phi}_{j^{\prime}}\right\rangle\right\} .
$$

It is possible that $\hat{\Omega}_{k}$ is not positive definite, but this problem is easily remedied (Ruppert and Matteson, 2015 [32]). Another rank correlation, Spearman's $\rho$, is similar and is omitted here. In the Supplementary Materials S5.4, we show that for Gaussian copulas, the difference between the $\log$ determinant of $\hat{\Omega}_{k}$ as estimated and of $\boldsymbol{\Omega}_{k}$ is $O p(J \sqrt{(\log J) / n})$.

Additionally for t-copulas with $\hat{\Omega}_{t, k}$, we apply pseudo-maximum likelihood to estimate the tail parameter $\nu_{k}>0$ by maximizing the log copula density 
$\sum_{i=1}^{n_{k}} \log \left[c_{t, k}\left\{\hat{F}_{1 k}\left(X_{i 1 k}\right), \ldots, \hat{F}_{J k}\left(X_{i J k}\right) \mid \hat{\boldsymbol{\Omega}}_{t, k}, \nu_{k}\right\}\right]$, with $\hat{F}_{j k}(x)=\sum_{i=1}^{n_{k}} \mathbb{1}\left\{X_{i j k} \leq x\right\} /\left(n_{k}+1\right)$. Marshal and Zeevi (2002 [24]) discussed maximum pseudo-likelihood estimation of t-copulas with applications to modeling extreme co-movements of financial assets.

\subsection{Marginal Density $f_{j k}$ Estimation}

We estimate the marginal density $f_{j k}$ of the projected scores $X_{\cdot j k}$ using kernel density estimation: $\hat{f}_{j k}\left(\hat{x}_{j}\right)=\frac{1}{n_{k} h_{j k}} \sum_{i=1}^{n_{k}} K\left(\frac{\left\langle x-X_{i \cdot k}, \hat{\phi}_{j}\right\rangle}{h_{j k}}\right)$, with $K$ the standard Gaussian kernel, $\hat{\phi}_{j}$ the estimated $j$-th joint eigenfunction, $h_{j k}=\hat{\sigma}_{j k} h$ the bandwidth for scores projected on $\hat{\phi}_{j}$ in group $k, \hat{\sigma}_{j k}$ as the estimated standard deviation of $\sigma_{j k}=\sqrt{\operatorname{Var}\left(X_{\cdot j k}\right)}$, and $\hat{x}_{j}=\left\langle x, \hat{\phi}_{j}\right\rangle$. Then $\log Q_{J}^{*}(x)$ in Eq.(2.5) is estimated by

$$
\log \hat{Q}_{J}^{*}(x)=\log \left(\frac{\hat{\pi}_{1}}{\hat{\pi}_{0}}\right)+\sum_{j=1}^{J} \log \left\{\frac{\hat{f}_{j 1}\left(\hat{x}_{j}\right)}{\hat{f}_{j 0}\left(\hat{x}_{j}\right)}\right\}+\log \left\{\frac{\hat{c}_{1}\left\{\hat{F}_{11}\left(\hat{x}_{1}\right), \ldots, \hat{F}_{J 1}\left(\hat{x}_{J}\right)\right\}}{\hat{c}_{0}\left\{\hat{F}_{10}\left(\hat{x}_{1}\right), \ldots, \hat{F}_{J 0}\left(\hat{x}_{J}\right)\right\}}\right\}
$$

where $\hat{c}_{k}$ is the Gaussian or t-copula density with estimated parameters, and $\hat{\pi}_{k}=n_{k} / n$. Proposition 1 in Section 5 shows that with an additional mild assumption, when the group eigenfunctions are unequal, $\left|\hat{f}_{j k}\left(\hat{x}_{j}\right)-f_{j k}\left(x_{j}\right)\right|$ is asymptotically bounded at the same rate as when eigenfunctions are equal. Detailed proofs are included in Supplementary Materials.

\subsection{Bayes Classifiers with Copula using Partial Least Squares}

An interesting alternative to principal components is functional partial least squares (FPLS). FPLS finds directions that maximize the covariance between the projected $X$ and $Y$ scores, rather than focusing on variation in $X$ alone as with PCA. As the algorithm in Supplementary Materials S1 describes, FPLS iteratively generates a weight function $w_{j}$ at each step $j, 1 \leq j \leq J$, which solves $\max _{w_{j} \in \mathcal{L}^{2}(\mathcal{T})} \operatorname{cov}^{2}\left\{Y^{j-1},\left\langle X^{j-1}, w_{j}\right\rangle\right\}$, such that $\left\|w_{j}\right\|=1$ and $\left\langle w_{j}, G\left(w_{j}^{\prime}\right)\right\rangle=0$ for all $1 \leq j^{\prime} \leq j-1$. Recall that $G$ is the joint covariance operator of the 
random function $X$. Here, $Y^{j-1}, X^{j-1}$ are the updated function $X$ and indicator $Y$ at step $j-1$ (see S1), and their corresponding sample values are noted as $Y_{i}^{j-1}, X_{i \cdot .}^{j-1}, i=1, \ldots, n$.

The algorithm gives the decomposition $X_{i . .}(t)=\sum_{j=1}^{J} s_{i j} P_{j}(t)+E_{i}(t), t \in \mathcal{T}$, where $\mathbf{s}_{i}=\left(s_{i 1}, \ldots, s_{i J}\right)^{T}$ is the length $J$ score vector, $P_{j} \in \mathcal{L}^{2}(\mathcal{T}), 1 \leq j \leq J$ are loading functions, and $E_{i}$ is the residual. Preda et al. (2007 [29]) investigated PLS in linear discriminant analysis (LDA), and defined score vectors $\mathbf{S}_{j}$ as eigenvectors of the product of the Escoufier's operators of $X$ and $Y$ (Escoufier, $1970[10]$ ). For our case, the classifiers BCG and BCt now can act on the PLS scores $\mathbf{s}_{i}=\left(s_{i 1}, \ldots, s_{i J}\right)^{T}$ of each observation $X_{i \ldots}$. We will refer to these classifiers as BCG-PLS and BCt-PLS.

The dominant PCA directions might only have large within-group variances and small between-group differences in means. Such directions will have little power to discriminate between groups. This problem can be fixed by FPLS. The advantages of FPLS have been discussed, for example, by Preda et al. (2007 [29]) and Delaigle and Hall (2012a [9]). The latter found that, when the difference between the group means projected on $j$-th PC direction is large only for large $j$, their functional centroid classifier with PLS scores has lower misclassification rates than using PCA scores. As later examples show, FPLS is especially effective in such situations.

\section{Comparison of Classifiers using Simulated Data}

\subsection{Data Design}

To set up the simulation, for simplicity, we use $\pi_{1}=\pi_{0}=0.5$. By Karhunen-Loève expansions, the functions $X_{i \cdot k}, i=1, \ldots, n_{k}$, of group $k=0,1$ can be decomposed as $X_{i \cdot k}=\mu_{k}+\sum_{j=1}^{J} \sqrt{\lambda_{j k}} \xi_{i j k} \phi_{j k}$, where $\mu_{k}$ is the group mean, $\lambda_{j k}$ is the $j$-th eigenvalue in group $k$ corresponding to eigenfunction $\phi_{j k}$, and $\lambda_{1 k}>\cdots>\lambda_{J k}$. The variables $\xi_{i j k}$ 
are distributed with $E\left(\xi_{i j k}\right)=0, \operatorname{var}\left(\xi_{i j k}\right)=1$ and $\operatorname{cov}\left(\xi_{i j k}, \xi_{i j^{\prime} k}\right)=0, \forall j \neq j^{\prime}$. The compact interval $\mathcal{T}$ is $[0,1]$, and the functions $X_{i \cdot k}$ are observed at the equally-spaced grid $t_{1}=0, t_{2}=1 / 50, \ldots, t_{51}=1$, with i.i.d. Gaussian noise $\epsilon_{i k}(t)$ centered at 0 and standard deviation 0.5. The classifiers are implemented both with and without pre-smoothing the data. As they have similar performances, we report only the results using pre-smoothing. The total sample size is $n=250,100$ training and 150 test cases. The number of eigenfunctions for curve generation is $J=201$, double the size of the training dataset, to imitate the infinite dimensions of functional data. For each $j$, the bandwidth $h_{j k}$ for KDE is selected by the direct plug-in method (Sheather and Jones, 1991 [34]). Simulations are repeated $N=1000$ times. Supplementary S3.1 also includes additional results with increased training size.

The distribution of $(X, Y)$ is determined by four factors: eigenfunctions (whether common or group-specific), difference between group means, eigenvalues, and score distributions. The factors are varied according to a $2 \times 2 \times 2 \times 3$ full factorial design described below. We adopt a four-letter system to label the 24 factor-level combinations, which we call "scenarios".

Factor 1: Eigenfunctions $\phi_{1 k}, \ldots, \phi_{J k}$ of group $k$ : The first factor specifies eigenfunctions of the covariance operators $G_{1}$ and $G_{0}$. When the two sets $\phi_{1 k}, \ldots, \phi_{J k}, k=0,1$, are the same, let the common eigenfunctions be the Fourier basis on $\mathcal{T}=[0,1]$, where $\phi_{1 k}(t)=1, \phi_{j k}(t)=\sqrt{2} \cos (j \pi t)$ or $\sqrt{2} \sin ((j-1) \pi t)$ for $1<j \leq 201$ even or odd.

When the two groups have unequal eigenfunctions, group $k=0$ uses the Fourier basis $\phi_{10}, \ldots, \phi_{J 0}$ as above, but group $k=1$ has a Fourier basis rotated by iterative updating:

i) let the starting value of $\phi_{11}, \ldots, \phi_{J 1}$ be the original Fourier basis functions as above;

ii) at step $\left(j, j^{\prime}\right)$ where $1 \leq j \leq J-1, j^{\prime}=j+1, \ldots, J$, the pair of functions $\left(\phi_{j 1}^{*}, \phi_{j^{\prime} 1}^{*}\right)$ is generated by a Givens rotation of angle $\theta_{j j^{\prime}}$ of the current pair $\left(\phi_{j 1}, \phi_{j^{\prime} 1}\right)$ such that 


$$
\phi_{j 1}^{*}(t)=\cos \left(\theta_{j j^{\prime}}\right) \phi_{j 1}(t)-\sin \left(\theta_{j j^{\prime}}\right) \phi_{j^{\prime} 1}(t), \phi_{j^{\prime} 1}^{*}(t)=\sin \left(\theta_{j j^{\prime}}\right) \phi_{j 1}(t)+\cos \left(\theta_{j j^{\prime}}\right) \phi_{j^{\prime} 1}(t) .
$$

iii) the rotation angle for each pair of $\left(j, j^{\prime}\right)$ is $\theta_{j j^{\prime}}=\frac{\pi}{3}\left(\lambda_{j 0}+\lambda_{j^{\prime} 0}\right)$, with $\lambda_{j 0}, \lambda_{j^{\prime} 0}$ the $j$-th and $j^{\prime}$-th eigenvalues of group $k=0$. Hence, the major eigenfunctions receive greater rotations, with the angles proportional to their eigenvalues;

iv) then we update $\phi_{j 1}, \phi_{j^{\prime} 1}$ with the new $\phi_{j 1}^{*}, \phi_{j^{\prime} 1}^{*}$ and continue the rotations until each pair of $\left(j, j^{\prime}\right)$ with $1 \leq j \leq J-1, j^{\prime}=j+1, \ldots, J$ is rotated.

The rotated Fourier basis of group $k=1$ guarantees that both groups $\Pi_{1}$ and $\Pi_{0}$ span the same eigenspace and satisfy the null hypothesis of the test of equal eigenspaces developed by Benko et al. (2009 [1]). This test was used by Dai et al. (2017 [7]) to check whether the two groups have the same eigenfunctions. However, having equal eigenspaces is a necessary, but not sufficient, condition for having equal sets of eigenfunctions, as proved by the rotated basis. Because of the unequal eigenfunctions of operators $G_{1}$ and $G_{0}$, scores $X_{i j k}$ will be correlated, which can be modeled by the new copula-based classifiers.

Other choices of the second set of eigenfunctions, including the Haar wavelet system on $\mathcal{L}^{2}([0,1])$, have also been tested, but with similar results and so are omitted. We denote the scenario where $\Pi_{1}$ and $\Pi_{0}$ have equal eigenfunctions as $S$ (same), otherwise as $R$ (rotated).

Factor 2: Difference, $\mu_{d}$, Between the Group Means: The second factor, which is at 2 levels, $\mathrm{S}$ (same) and D (different), is the difference between the group means, $\mu_{d}=\mu_{1}-\mu_{0}$. For simplicity, we let $\mu_{0}=0, \mu_{1}=\mu_{d}$. Here $\mu_{d}(t)=t$.

Factor 3: Eigenvalues $\lambda_{1 k}, \ldots, \lambda_{J k}$ of Group $k$ : The third factor, at two levels labeled $\mathrm{S}$ and $\mathrm{D}$, is whether eigenvalues $\lambda_{1 k}, \ldots, \lambda_{J k}$ depend on $k$. We label the level where $\lambda_{j 1}=$ $\lambda_{j 0}=1 / j^{2}$ as $\mathrm{S}$, and label the level when $\lambda_{j 1}=1 / j^{3}$ and $\lambda_{j 0}=1 / j^{2}$ as $\mathrm{D}, 1 \leq j \leq J$. 
Factor 4: Distribution of the standardized scores $\xi_{i j k}$ : The fourth factor, at three levels $\mathrm{N}$ (normal), $\mathrm{T}$ (tail dependence and skewness), $\mathrm{V}$ (varied), is the distribution of $\xi_{i j k}$. $N: \xi_{i 1 k}, \ldots, \xi_{i J k}$ have Gaussian distribution $N(0,1)$ for both $k=0$ and 1.

T: This level includes tail dependency by setting $\xi_{i j k}=\left(\delta_{i j k}-b\right) / \eta_{i k}$, where $\delta_{i j k} \sim$ $\operatorname{Exp}\left(\lambda^{*}\right), \lambda^{*}=5 \sqrt{3} / 3, b=1 / \lambda^{*}$, and $\eta_{i k} \sim \chi^{2}(5) / 5$ for all $j=1, \ldots, J$. All of $\delta_{i j k}$ and $\eta_{i k}$ are mutually independent, while the scores $\xi_{i j k}$ on each basis $j$ are uncorrelated but dependent, as they share the same denominator, $\eta_{i k}$. The scores are skewed in both groups.

$V$ : In this level, the scores in the two groups have different types of distributions, with $\xi_{i j 1} \sim N(0,1), \xi_{i j 0} \sim \operatorname{Exp}(1)-1$. Simulation results of a different choice of the varied distributions of $\xi_{i j 1}$ and $\xi_{i j 0}$ are included in Supplementary Materials Section S3.1 Table S1.

Table 1 lists all 24 scenarios used in the simulations:

\begin{tabular}{|c|c|c|c|}
\hline & $\xi_{i j k} \sim \mathrm{N}$ & $\xi_{i j k} \sim \mathrm{T}$ & $\xi_{i j k} \sim \mathrm{V}$ \\
\hline$\mu_{d}=0, \lambda_{j 1}=\lambda_{j 0}$ & $(\mathrm{R} / \mathrm{S}) \mathrm{SSN}$ & $(\mathrm{R} / \mathrm{S}) \mathrm{SST}$ & $(\mathrm{R} / \mathrm{S}) \mathrm{SSV}$ \\
\hline$\mu_{d}=0, \lambda_{j 1} \neq \lambda_{j 0}$ & $(\mathrm{R} / \mathrm{S}) \mathrm{SDN}$ & $(\mathrm{R} / \mathrm{S}) \mathrm{SDT}$ & $(\mathrm{R} / \mathrm{S}) \mathrm{SDV}$ \\
\hline$\mu_{d} \neq 0, \lambda_{j 1}=\lambda_{j 0}$ & $(\mathrm{R} / \mathrm{S}) \mathrm{DSN}$ & $(\mathrm{R} / \mathrm{S}) \mathrm{DST}$ & $(\mathrm{R} / \mathrm{S}) \mathrm{DSV}$ \\
\hline$\mu_{d} \neq 0, \lambda_{j 1} \neq \lambda_{j 0}$ & $(\mathrm{R} / \mathrm{S}) \mathrm{DDN}$ & $(\mathrm{R} / \mathrm{S}) \mathrm{DDT}$ & $(\mathrm{R} / \mathrm{S}) \mathrm{DDV}$ \\
\hline
\end{tabular}

Table 1: Simulation scenarios. The labels are ordered: eigenfunctions (R/S), group mean (S, D), eigenvalues $(\mathrm{S}, \mathrm{D})$, and $\xi_{i j k}$ distributions $(\mathrm{N}, \mathrm{T}, \mathrm{V})$. Note that in SSSN and SSST functions from both groups have the same distribution. We simply include them to have a full factorial design.

\subsection{Functional Classifiers}

Classifiers in this study are listed below. Five of them are Bayes classifiers, while the last three are non-Bayes. Methods proposed in this paper are described in (ii) - (iii).

(i) BC: the original Bayes classifier of Dai et al. (2017 [7]), whose log density ratio is given by Eq.(2.2). The scores are by projection onto principal components (PC);

(ii) BCG, BCG-PLS: the Bayes classifiers with a Gaussian copula to model correlation, 
using PC and PLS scores respectively. Rank correlation used is Kendall's $\tau$. Both Gaussian and t-copula densities can be implemented using the $\mathrm{R}$ package copula [15];

(iii) BCt, BCt-PLS: the Bayes classifiers similar to (ii), but using a t-copula instead;

(iv) CEN: functional centroid classifier in Delaigle and Hall (2012a [9]), where observation $x$ is classified to group $k=1$, if $T(x)=\left(\langle x, \psi\rangle-\left\langle\mu_{1}, \psi\right\rangle\right)^{2}-\left(\langle x, \psi\rangle-\left\langle\mu_{0}, \psi\right\rangle\right)^{2} \leq 0$, with $\mu_{1}, \mu_{0}$ the group means. Here $\psi=\sum_{j=1}^{J^{*}} \lambda_{j}^{-1} \mu_{j} \phi_{j}$ is a function of first $J^{*}$ joint eigenfunctions $\phi_{j}$, the corresponding eigenvalues $\lambda_{j}$, and $\mu_{j}=\left\langle\mu_{1}-\mu_{0}, \phi_{j}\right\rangle$;

(v) PLSDA (PLS Discriminant Analysis): binary classifier using Fisher's linear discriminant rule with FPLS as a dimension reduction method. It is implemented in the $\mathrm{R}$ package pls [27];

(vi) Logistic regression: logistic regression on functional principal components implemented by the $\mathrm{R}$ function glm . It is one of the functional generalized regressions discussed in Müller and Stadtmüller (2005) [28].

In each simulation, $J^{*}$ is selected by 10 -fold cross validation on training data. The candidate $J$ values range from 1 to 30 (2 to 30 for classifiers using copulas). Estimation of joint eigenfunctions $\phi_{j}$ follows the discretization approach to functional principal components analysis, as described in Chapter 8.4 of Ramsay and Silverman (2005 [30]). Similar discretization strategy is used for PLS basis.

\subsection{Classifier Performances}

Table 2 contains the average misclassification rate over 1000 simulations by each method on each scenario. In addition, for each simulation we use 10-fold cross validation to select the classifier with the best performance on training data among the eight classifiers in Section 3.2. 


\begin{tabular}{r|r|rrrr|rrr|rr}
\hline & BC & BCG & BCGPLS & BCt & BCtPLS & CEN & PLSDA & logistic & CV & Ratio (CV) \\
\hline SSSN & 0.502 & 0.502 & 0.500 & 0.500 & 0.501 & 0.502 & 0.501 & 0.500 & 0.501 & $0.23 \%$ \\
SSDN & 0.227 & 0.244 & 0.345 & 0.258 & 0.443 & 0.464 & 0.495 & 0.466 & 0.232 & $2.43 \%$ \\
SDSN & 0.347 & 0.351 & 0.361 & 0.351 & 0.363 & 0.275 & 0.304 & 0.279 & 0.291 & $5.88 \%$ \\
SDDN & 0.169 & 0.173 & 0.303 & 0.175 & 0.327 & 0.231 & 0.262 & 0.234 & 0.173 & $2.64 \%$ \\
\hline SSST & 0.507 & 0.502 & 0.500 & 0.505 & 0.499 & 0.499 & 0.499 & 0.499 & 0.502 & $0.69 \%$ \\
SSDT & 0.438 & 0.441 & 0.454 & 0.456 & 0.471 & 0.488 & 0.497 & 0.490 & 0.452 & $3.19 \%$ \\
SDST & 0.188 & 0.183 & 0.270 & 0.184 & 0.311 & 0.167 & 0.234 & 0.169 & 0.170 & $1.96 \%$ \\
SDDT & 0.166 & 0.161 & 0.237 & 0.160 & 0.296 & 0.148 & 0.233 & 0.150 & 0.152 & $2.59 \%$ \\
\hline SSSV & 0.355 & 0.361 & 0.484 & 0.363 & 0.493 & 0.476 & 0.481 & 0.489 & 0.363 & $2.20 \%$ \\
SSDV & 0.253 & 0.270 & 0.373 & 0.276 & 0.430 & 0.455 & 0.477 & 0.462 & 0.257 & $1.78 \%$ \\
SDSV & 0.264 & 0.275 & 0.401 & 0.276 & 0.408 & 0.279 & 0.315 & 0.283 & 0.273 & $3.27 \%$ \\
SDDV & 0.202 & 0.209 & 0.309 & 0.207 & 0.313 & 0.236 & 0.280 & 0.238 & 0.210 & $3.95 \%$ \\
\hline RSSN & 0.327 & 0.147 & 0.183 & 0.147 & 0.180 & 0.494 & 0.497 & 0.485 & 0.151 & $2.67 \%$ \\
RSDN & 0.252 & 0.090 & 0.140 & 0.093 & 0.164 & 0.489 & 0.500 & 0.482 & 0.093 & $2.93 \%$ \\
RDSN & 0.287 & 0.128 & 0.154 & 0.128 & 0.152 & 0.327 & 0.333 & 0.329 & 0.131 & $2.71 \%$ \\
RDDN & 0.208 & 0.077 & 0.112 & 0.079 & 0.128 & 0.287 & 0.300 & 0.288 & 0.080 & $3.44 \%$ \\
\hline RSST & 0.435 & 0.354 & 0.373 & 0.357 & 0.372 & 0.486 & 0.490 & 0.489 & 0.361 & $1.95 \%$ \\
RSDT & 0.400 & 0.326 & 0.348 & 0.336 & 0.365 & 0.486 & 0.491 & 0.485 & 0.339 & $3.87 \%$ \\
RDST & 0.178 & 0.148 & 0.248 & 0.154 & 0.261 & 0.174 & 0.252 & 0.175 & 0.156 & $5.80 \%$ \\
RDDT & 0.166 & 0.137 & 0.217 & 0.142 & 0.255 & 0.159 & 0.249 & 0.158 & 0.147 & $7.68 \%$ \\
\hline RSSV & 0.266 & 0.147 & 0.202 & 0.149 & 0.204 & 0.472 & 0.481 & 0.475 & 0.150 & $1.71 \%$ \\
RSDV & 0.233 & 0.100 & 0.143 & 0.105 & 0.157 & 0.465 & 0.475 & 0.469 & 0.104 & $3.85 \%$ \\
RDSV & 0.241 & 0.145 & 0.183 & 0.146 & 0.191 & 0.332 & 0.349 & 0.337 & 0.148 & $2.28 \%$ \\
RDDV & 0.238 & 0.116 & 0.157 & 0.120 & 0.167 & 0.299 & 0.325 & 0.300 & 0.121 & $3.97 \%$ \\
\hline
\end{tabular}

Table 2: Misclassification rates of eight classifiers on 24 scenarios, each an average from 1000 simulations. Lowest rates of each data case are colored in dark green, and cases within margin of error (see text) of the lowest are colored in light green. The column labeled CV contains error rates of the classifier selected by cross validation. Ratio $(\mathrm{CV})$ is the percent difference from the best of the eight classifiers for that scenario. CV error rates are not included in the rankings that determine coloring. SSSN and SSST are colored gray, as there is actually no difference between groups in these scenarios, and, since $\pi_{0}=\pi_{1}=1 / 2$, the true misclassification rate is 0.5 .

Average misclassification rates of the CV-selected classifier are listed in the CV column. The column Ratio(CV) contains the percentage difference between the CV-selected (CV) and best (opt) classifier: Ratio $(\mathrm{CV})=\{\operatorname{err}(\mathrm{CV})-\operatorname{err}(\mathrm{opt})\} / \operatorname{err}(\mathrm{opt}) \times 100 \%$. For each scenario, the lowest error rates of the eight classifiers are colored in dark green. We also use light green to label the ones within the optimal case's margin of error (MOE) for each data scenario $\gamma$ : $\mathrm{MOE}_{\gamma}=1.96 \times \sigma_{\gamma}^{*} / \sqrt{1000}$, where $\sigma_{\gamma}^{*}$ is the sample standard deviation of the best classifier (at scenario $\gamma$ )'s error rates from 1000 simulations. The simulations enable a comprehensive understanding of the classifiers' behaviors, which we now discuss. 
- Equal versus Unequal Eigenfunctions. Comparison between the top and bottom half of Table 2 demonstrates the strength of our copula-based classifiers, especially on unequal eigenfunctions (bottom half). By its nature, $\mathrm{BC}$ has strong performance when the two groups have the same set of eigenfunctions and the scores $\xi_{i j k}$ are mutually independent, e.g., in SSDN and SSDV. However, when the data have more complicated structure like score tail dependency and location difference, CEN and logistic get better results (SDST, SDDT). It is worth noting that in every case with equal eigenfunctions, $\mathrm{BCG} / \mathrm{BCt}$ are always the ones with rates closest to BC's.
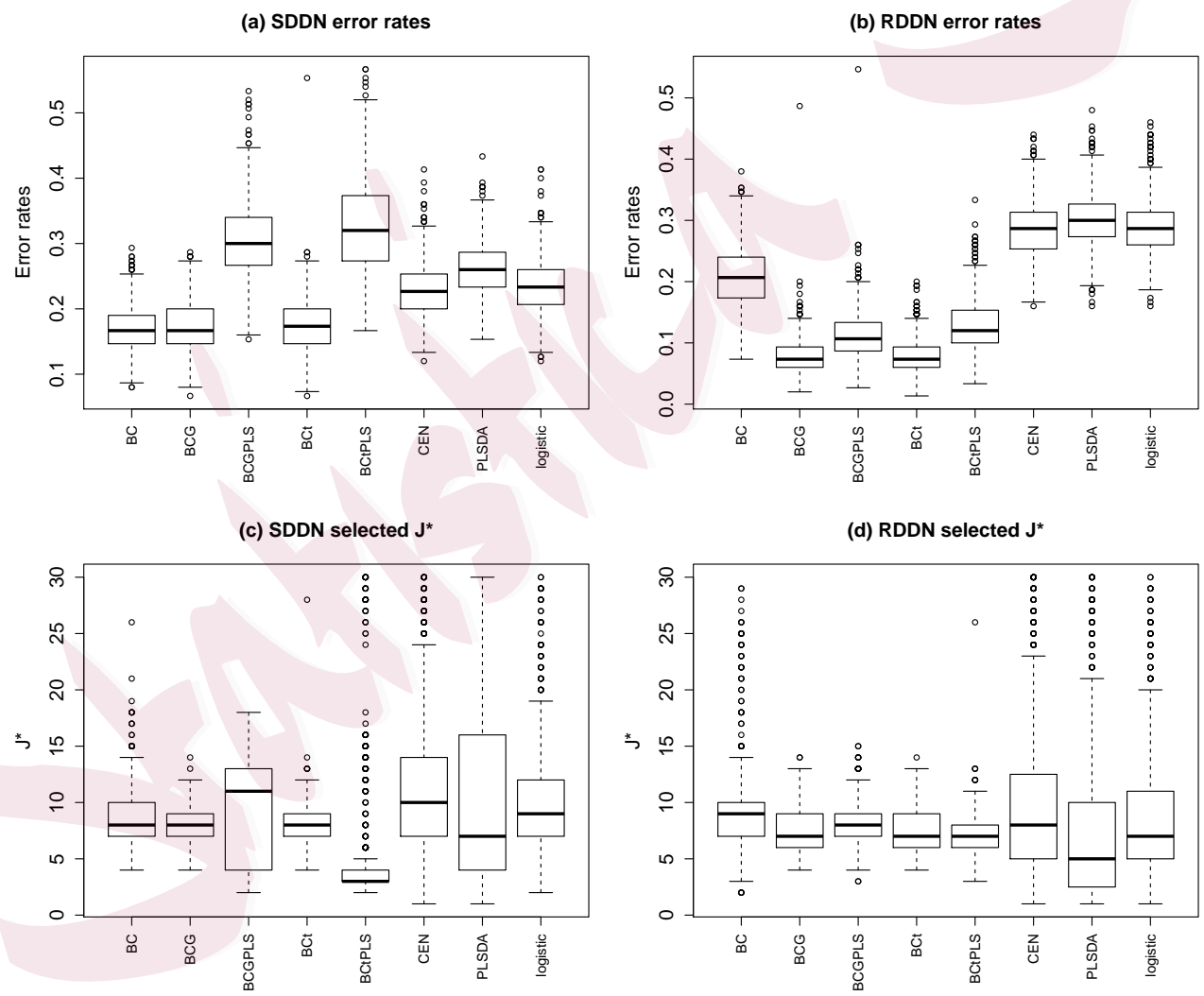

Figure 2: Part (a) and (b) are boxplots of error rates by the eight classifiers in scenarios SDDN and RDDN. The bottom two plots (c) and (d) are boxplots of cross-validated $J^{*}$ correspondingly in each simulation.

On the other hand, when the group eigenfunctions are different, $\mathrm{BC}$ and the three non-Bayes classifiers fail to outperform BCG/BCt in any scenario, even though the 
group eigenspaces remain equal. BCG keeps its robust performance of lowest error rates throughout all cases, while BCt is not far behind, and is able to fall into BCG's MOE $50 \%$ of the times as labeled.

Fig. 2 compares misclassification rates and the corresponding $J^{*}$ selected in each of the 1000 simulations at two scenarios SDDN and RDDN. These two scenarios differ only in eigenfunction setting. In Plot (a) where the groups have equal eigenfunctions, BC, BCG and BCt show similar behaviors in classification. In Plot (b) where the group eigenfunctions differ, BCG and BCt have lowest error rates and variation, followed by BCG-PLS and BCt-PLS. In Plots (c) and (d), we find that BCG and BCt are the only classifiers that have stable choice of optimal $J^{*}$ : both methods choose $J^{*}<10$ for more than $75 \%$ of the times with few outliers, group eigenfunctions equal or not.

- Difference between the group means. Under equal eigenfunction setting, non-Bayes classifiers like CEN and logistic regression are naturally sensitive to location difference, especially when other factors are kept the same, e.g. SDSN, SDST. However, in the bottom half of Table 2 where the group eigenfunctions differ, BCG shows strongest performance in all cases, with BCt a close second.

In this table, PC based methods BCG and BCt show advantage over their PLS counterparts in scenarios with location difference. That is because $\mu_{d}$ here is effectively captured by principal components. In Section 3.4, when the new $\mu_{d}$ has nonzero projections only on the last several basis, PLS based classifiers can do a better job than other methods in distinguishing such difference, as mentioned in Delaigle and Hall (2012a [9]). This phenomenon is also discussed in Section 4. 
- Difference in group eigenvalues and score distributions. In general, we find that the marginal densities of the scores as well as their eigenvalues have similar impact on classifiers' performance. They contribute to the difference of functional distributions in each group, which the three non-Bayes methods (CEN, PLSDA, logistic) fail to detect. For all scenarios in Table 2 without location difference, CEN, PLSDA and logistic regression all show very poor performance with error rates close to $50 \%$.

The two right-most columns in Table 2 show that the CV-selected method achieves comparable performance to the optimal result of each scenario. It demonstrates the stability and strength of our copula-based Bayes classifiers, especially under the unequal eigenfunction setting. Sections S3.2 and S3.3 in the Supplementary Materials report the correlations between the first ten scores in scenarios RSDN and RSDT, respectively. These high correlations are consistent with the strong performance of the copula-bases classifiers in scenarios where the two groups have different eigenfunctions.

\subsection{Multiclass Classification Performance}

We also investigate performance of aforementioned methods on classifying data into more than two labels, as the group eigenfunctions from multiple different classes are more likely to be unequal, and the necessity increases to consider dependency of scores on the joint basis.

We now denote the group labels as $Y=k, k=0,1,2$, and set up the multiclass scenarios following the design in Section 3.1. The first column in Table 3 lists 12 scenarios considered. The first letter $M$ labels unequal group eigenfunctions: when $Y=0$ and 1, the group eigenfunctions are respectively Fourier basis and its rotated counterpart as described in type R of Factor 1 for binary data; when $Y=2$, the group basis is again rotated Fourier functions on $\mathcal{T}=[0,1]$, but the rotation angle factor used in iii) of Factor 1 in Section 3.1 is now $\pi / 4$ 
instead of $\pi / 3$. We omit cases of equal group eigenfunctions, as similar results can be found in the binary setup, and the likelihood of unequal basis increases as the levels of $Y$ go up.

The second letter $\mathrm{S}$ or $\mathrm{D}$ again denotes equal group means or not. When the group means $\mu_{k}$ are unequal (labeled D), we set $\mu_{0}=0, \mu_{1}$ the identity function used previously, and $\mu_{2}=\sum_{j=192}^{201} \phi_{j 0}$. Function $\mu_{2}$ follows similar design of Delaigle and Hall (2012a [9]), where the group mean only has nonzero weights on the last 3 of 40 eigenfunctions. We here assign the nonzero weights to the last 10 of 201 basis.

Similarly, S or D in the third position represents same or different group eigenvalues. When group eigenvalues are equal, $\lambda_{j k}=10 / j^{2}$ for all $k$; otherwise $\lambda_{j k}=10 / j^{2}, 10 / j^{3}, 10 / j$ respectively for $k=0,1,2, j \geq 1$. Finally, the last letter inherits the design from Factor 4 of Section 3.1 to describe the standardized score distribution patterns: similar to the binary case, $\mathrm{N}$ and $\mathrm{T}$ stands for the Gaussian and skewed distributions for all three levels, while for $\mathrm{V}$ we define scores $\epsilon_{i j k}$ to follow either standard Gaussian, centered Exponential with rate 1 , or the skewed distribution in $\mathrm{T}$ for $k=0,1,2$ respectively.

The other setup details of noise, data pre-smoothing, bandwidth selection are all similar to Section 3.1 for binary data. For each simulation, we have 100 training and 150 test cases. The optimal cut-off $J^{*}$ is selected by cross validation from $J \leq 10$. Table 3 presents misclassification rates from 1000 Monte Carlo repetitions, by 7 of the 8 classifiers in Section 3.2. Note that functional centroid classifier is not applicable to multiclass data and thus is excluded here. Like the binary case, we include in the Supplementary Materials Table S2 additional results with increased training size and a different set of score distributions (V).

Table 3 indicates that for data of multiple labels, behaviors of the 7 classifiers follow a similar pattern of the binary case when group eigenfunctions are unequal. Especially, 


\begin{tabular}{r|r|rrrr|rr|rr}
\hline & BC & BCG & BCGPLS & BCt & BCtPLS & PLSDA & logistic & CV & Ratio(CV) \\
\hline MSSN & 0.520 & 0.325 & 0.392 & 0.327 & 0.392 & 0.641 & 0.637 & 0.328 & $0.89 \%$ \\
MDSN & 0.356 & 0.247 & 0.237 & 0.245 & 0.235 & 0.446 & 0.427 & 0.226 & $-3.88 \%$ \\
MSDN & 0.213 & 0.169 & 0.281 & 0.168 & 0.310 & 0.636 & 0.618 & 0.173 & $3.00 \%$ \\
MDDN & 0.194 & 0.156 & 0.272 & 0.156 & 0.295 & 0.540 & 0.509 & 0.157 & $1.11 \%$ \\
\hline MSST & 0.560 & 0.450 & 0.503 & 0.450 & 0.492 & 0.635 & 0.638 & 0.456 & $1.25 \%$ \\
MDST & 0.343 & 0.286 & 0.303 & 0.286 & 0.333 & 0.424 & 0.364 & 0.284 & $-0.72 \%$ \\
MSDT & 0.449 & 0.399 & 0.444 & 0.397 & 0.467 & 0.624 & 0.616 & 0.401 & $0.95 \%$ \\
MDDT & 0.342 & 0.297 & 0.355 & 0.287 & 0.403 & 0.483 & 0.401 & 0.293 & $2.38 \%$ \\
\hline MSSV & 0.325 & 0.259 & 0.394 & 0.261 & 0.475 & 0.633 & 0.615 & 0.264 & $2.23 \%$ \\
MDSV & 0.288 & 0.237 & 0.356 & 0.234 & 0.433 & 0.436 & 0.399 & 0.241 & $2.93 \%$ \\
MSDV & 0.385 & 0.314 & 0.427 & 0.302 & 0.435 & 0.631 & 0.627 & 0.311 & $3.00 \%$ \\
MDDV & 0.272 & 0.223 & 0.322 & 0.219 & 0.340 & 0.475 & 0.434 & 0.224 & $2.18 \%$ \\
\hline
\end{tabular}

Table 3: Misclassification rates averaged over 1000 simulations of the 7 classifiers on 12 multiclass data scenarios. Best case in each scenario is colored in dark green, and cases within margin of error of the lowest are colored in light green. $P(Y=k)=1 / 3$ for $k=0,1,2$, so the true misclassification rate of any method is approximately 0.667 .

BCt shows strength under increased data complexity, with BCG closely following. BCGPLS/BCt-PLS also prove their advantage in detecting location difference on minor basis functions in MDSN. Although they fail to outperform their PC-based counterparts under more complicated scenarios like MDST and MDSV, we believe it is because group means are not the only dominant difference in these two data cases.

Table 2 and 3 give us clear guidelines that, whether or not to use copulas in classification makes a more significant impact on the outcome than the type of copulas, since both BCG and BCt present competitive performance. They also reveal the strength of copula based methods in dimension reduction. Classifiers using copulas are able to achieve high accuracy with small cut-off $J^{*}$, which indicates their advantage in data of small sample size. Also, in general, principal components are preferable over PLS due to their robustness and simplicity of implementation. BCG-PLS and BCt-PLS should be considered when the group mean difference is significant and located at minor eigenfunctions, which we will discuss more in the real data examples. 


\section{Real Data Examples}

In this section, we use two real data examples to illustrate the strength of our new method in classification as well as dimension reduction with respect to data size $n$.

\subsection{Classification of Multiple Sclerosis Patients}

Our first real data example explores the classification of multiple sclerosis (MS) cases based on fractional anisotropy (FA) profiles of the corpus callosum (cca) tract. Fractional anisotropy (FA) is the degree of anisotropy of water diffusion along a tract and is measured by diffusion tensor imaging (DTI). Outside the brain, water diffusion is isotropic (Goldsmith et al., 2012 [13]). MS is an autoimmune disease leading to lesions in white matter tracts such as the corpus callosum. These lesions decrease FA.

The DTI dataset in the $\mathrm{R}$ package refund [14] contains FA profiles at 93 locations on the corpus callosum of 142 subjects. The data were collected at Johns Hopkins University and the Kennedy-Krieger Institute. The numbers of visits per subject range from 1 to 8, but we used only the 142 FA curves from first visits. One subject with partially missing FA data was removed. Among the 141 subjects, 42 are healthy $(k=0)$ and 99 were diagnosed with MS $(k=1)$. We use local linear regression for data pre-smoothing. To determine the optimal number of dimensions $J^{*}$ for each method, we use cross validation with maximal $J=30$. Misclassification rates by 10-fold cross-validation were recorded for 1000 repetitions.

As discussed in Section 1, Panel (a) in Fig. 1 plots 5 FA profiles from each group, and panels (b) and (c) display the group means and standard deviations of cases and controls, using raw and pre-smoothed data. Compared to controls, MS patients have lower mean FA values and greater variability. We see that smoothing removes some noise. 


\begin{tabular}{r|rrrrrrrr}
\hline Method & BC & BCG & BCGPLS & BCt & BCtPLS & CEN & PLSDA & logistic \\
\hline Error Rate & 0.228 & 0.199 & 0.211 & 0.192 & 0.211 & 0.264 & 0.219 & 0.216 \\
\hline
\end{tabular}

Table 4: Average misclassification rates of eight functional classifiers by 1000 repetitions of 10 -fold CV. BCt has the best performance. The best case is colored dark green.

(a) error rates of MS classification

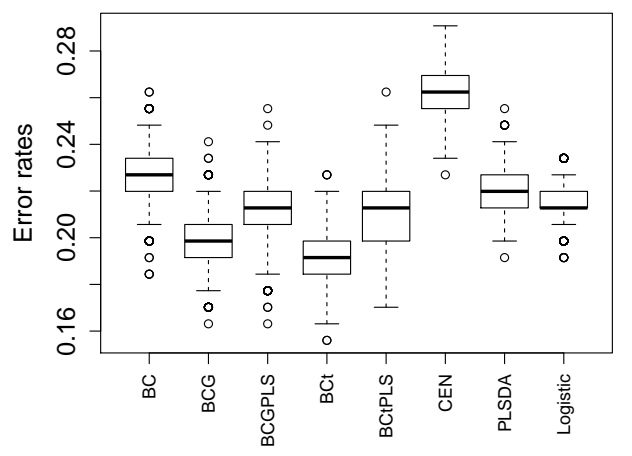

(b) optimal J* selected

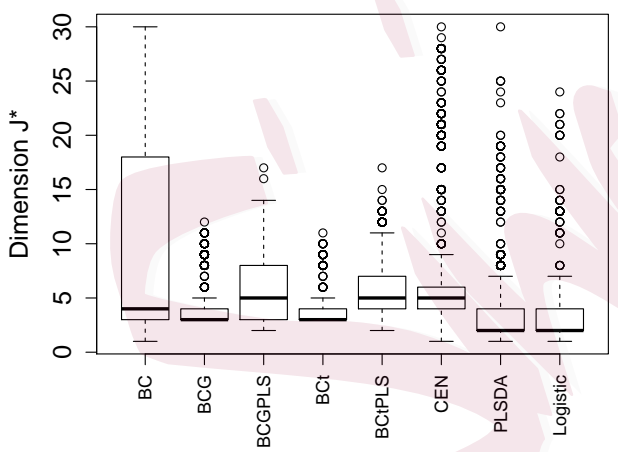

Figure 3: Boxplots of misclassification rates and optimal number of components $J^{*}$ in the MS study over 1000 repetitions of 10-fold cross-validation. BCt achieves the lowest average error rate, while requiring a very small number of components $\left(J^{*}<5\right)$ with lowest variation.

As shown in Table 4 and Part (a) of Fig. 3, BCt achieves the lowest error rate at 0.192, with a margin of error 0.0007 . Rates by other methods fail to fall into this range, and are all significantly higher than BCt's. In fact, the third quartile for BCt is below the first quartile of all other methods except BCG. Part (b) is a boxplot of cross validated $J^{*}$ during each simulation for all classifiers. Here BCt and BCG show their ability to achieve the lowest error rates with a minimal number of dimensions. In addition, compared to other methods like CEN, PLSDA or logistic regression, their choice of optimal $J^{*}$ is very stable, with smallest variation and few outliers. In contrast, $\mathrm{BC}$ is prone to employ a large number of components in classification. Such tendency can be found in other examples too.

In the Supplementary Materials, we compare the loadings (S3), score distributions (S5 and group eigenfunctions (S4) between using PC and PLS. The difference explains why PC is a better choice for this example. Note that it is not our intent to develop DTI as a technique 
for diagnosing MS. DTI is too expensive and time-consuming for that purpose. Instead, we are looking for differences in FA between cases and controls, since these could inform researchers about the nature of the disease. We have found clear differences between cases and controls in the mean and variance of FA. The strong positive correlation between second and third principal component scores in the healthy cases (Spearman's $\rho$ at 0.525 and an adjusted $p$-value $2 \times 10^{-2}$ ) is diminished in MS group. BCt as well as BCG is best able to use a compact model to capture subtle differences such as in correlations here.

\subsection{Particulate Matter (PM) Emission of Heavy Duty Trucks}

As a second example, we investigate the relationship between movement patterns of heavy duty trucks and particulate matter (PM) emissions. We use the data in McLean et al. (2015 [25]) originally extracted from the Coordinating Research Council E55/59 emissions inventory program documentary (Clark et al. 2007 [4]). The dataset contains 108 records of truck speed in miles/hour over 90 second intervals, and the logarithms of their PM emission in grams (log PM), captured by $70 \mathrm{~mm}$ filters.

We dichotomize log PM. The 41 of 108 cases with log PM above average are called high emission $(k=1)$ and the other cases are low emission $(k=0)$. We classify log PM level using the 90 -second velocity profiles. Misclassifications rates were estimated using 10-fold cross validation repeated 1000 times.

As Fig. 4 shows, during the first 20 seconds, vehicles in high PM group on average decelerate to a minimum speed, while the low PM group tends to speed up. The high PM group also has much lower variation than the low PM group.

From Fig. 5 and Table 5, BCG-PLS and BCt-PLS have the lowest misclassification rates. The third quartiles of their error rates are below first quartiles of the other classifiers except 

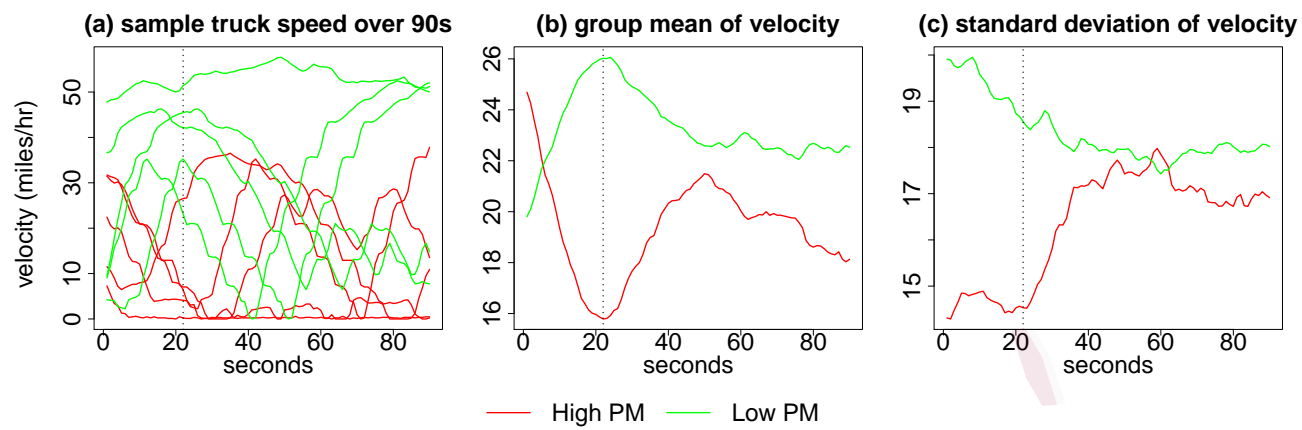

Figure 4: Plots of five sample paths in each PM group, as well as group mean and standard deviation of truck velocity data. On average, trucks in high PM group have lowest speed at 22 seconds, marked with a dashed line on each plot.

\begin{tabular}{l|rrrrrrrr}
\hline & BC & BCG & BCGPLS & BCt & BCtPLS & CEN & PLSDA & logistic \\
\hline Error rate & 0.285 & 0.280 & 0.207 & 0.280 & 0.207 & 0.278 & 0.256 & 0.228 \\
\hline
\end{tabular}

Table 5: Average misclassification rates of eight functional classifiers by 1000 repetitions of 10-fold CV. BCt-PLS and BCG-PLS have the best performance. The best cases are colored dark green.

(a) error rates of truck classification

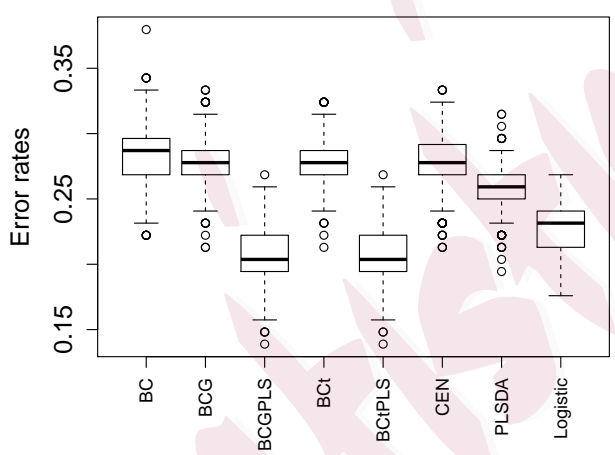

(b) optimal $\mathrm{J}^{\star}$ selected

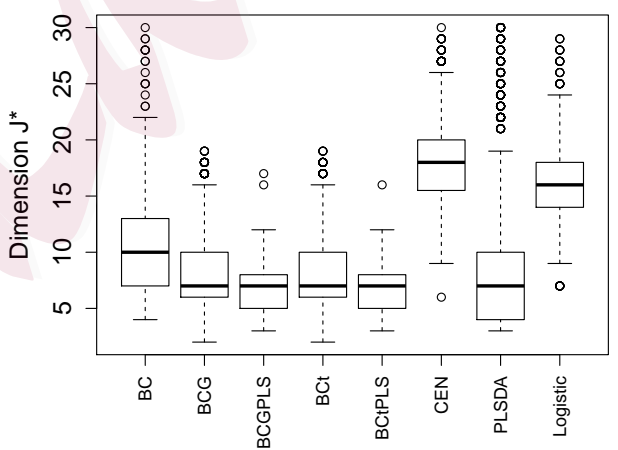

Figure 5: Boxplots of misclassification rates and optimal number of components $J^{*}$ in the truck emission case over 1000 repetitions of 10-fold cross-validation. BCt-PLS and BCG-PLS achieve the lowest average error rate with $J^{*}$ concentrated around 7 .

logistic regression. Also, both methods keep the classification model compact by requiring small $J^{*}$ with low variation. $\mathrm{BC}$ and the three methods on the right of plot (b) of Fig. 5 again demand more components with bigger variation in classification. In Section S4 of Supplementary Materials, we include additional results of both data examples to validate their different choices of PC and PLS based classifiers. 


\section{Theoretical Asymptotic Properties}

An interesting feature of functional classifiers is asymptotic perfect classification, i.e., under certain conditions, the error rate goes to 0 as $J \rightarrow \infty$, due to the infinite dimensional nature of functional data (Delaigle and Hall, 2012a [9]). Dai et al. (2017 [7]) discussed perfect classification by the functional Bayes classifier (BC) under equal group eigenfunctions. In this section, we prove that when the group eigenfunctions differ, perfect classification is retained by our classifier $\mathbb{1}\left\{\log Q_{J}^{*}(X)>0\right\}$ for both Gaussian and non-Gaussian processes. The scores $X_{\cdot j k}, 1 \leq j \leq J$ in this section are all projected on joint eigenfunctions $\phi_{1}, \ldots, \phi_{J}$.

We first show that $\log Q_{J}^{*}(X)$ and the estimated $\log \hat{Q}_{J}^{*}(X)$ are asymptotically equivalent under mild conditions. Then, the behavior of the Bayes classifier $\mathbb{1}\left\{\log Q_{J}^{*}(X)>0\right\}$ is studied in two settings: first, when the random function $X_{. . k}$ is a Gaussian process for both $k=0,1$; and second, the more general case when $X$ is non-Gaussian but its projected scores are meta-Gaussian distributed in each group. For simplicity, we assume here that $\pi_{1}=\pi_{0}$.

\subsection{Asymptotic equivalence of $\log \hat{Q}_{J}^{*}(X)$ and $\log Q_{J}^{*}(X)$}

We first list several assumptions, which help establish the asymptotic equivalence of both the marginal and copula density components of $\log \hat{Q}_{J}^{*}(X)$ and $\log Q_{J}^{*}(X)$.

Assumption A1. For all $C>0$ and some $\delta>0: \sup _{t \in \mathcal{T}} E\left\{|X(t)|^{C}\right\}<\infty$, $\sup _{s, t \in \mathcal{T}: s \neq t} E\left[\left\{|s-t|^{-\delta}|X(s)-X(t)|\right\}^{C}\right]<\infty$

Assumption A2. For integers $r \geq 1, \lambda_{j}^{-r} E\left[\int_{\mathcal{T}}\{X-E(X)\} \phi_{j}\right]^{2 r}$ is bounded uniformly in $j$. Assumption A3. There are no ties among the eigenvalues $\left\{\lambda_{j}\right\}_{j=1}^{\infty}$.

Assumption A4. The density $g_{j}$ of the $j$-th standardized score $\left\langle X-E(X), \phi_{j}\right\rangle / \sqrt{\lambda_{j}}$ is bounded and has a bounded derivative; for some $\delta>0, h=h(n)=O\left(n^{-\delta}\right)$ and $n^{1-\delta} h^{3}$ is 
bounded away from zero as $n \rightarrow \infty$. The ratio $f_{j 1}\left(X_{\cdot j .}\right) / f_{j 0}\left(X_{. j .}\right)$ is atomless for all $j \geq 1$.

For all $c>0$, let $\mathcal{S}(c)=\left\{x \in \mathcal{L}^{2}(\mathcal{T}):\|x\| \leq c\right\}$. Assumptions A1 - A4 are from Delaigle and Hall (2010 [8]), adapted here to bound the difference $D_{j k}\left(x_{j}\right)=\hat{g}_{j k}\left(\hat{x}_{j}\right)-\bar{g}_{j k}\left(x_{j}\right)$ s.t. $\sup _{x \in \mathcal{S}(c)}\left|D_{j k}\left(x_{j}\right)\right|=o p\left\{(n h)^{-1 / 2}\right\}$. We let $\hat{g}_{j k}\left(\hat{x}_{j}\right)=1 /\left(n_{k} h\right) \sum_{i=1}^{n_{k}} K\left\{\left\langle X_{i \cdot k}-x, \hat{\phi}_{j}\right\rangle /\left(\hat{\sigma}_{j k} h\right)\right\}$ be the estimated density of the standardized scores of group $k$ on basis $\hat{\phi}_{j}$, with $\bar{g}_{j k}\left(x_{j}\right)$ using $\phi_{j}$ and $\sigma_{j k}$. Also, the following assumption is added for $D_{j k}\left(x_{j}\right)$, for both $k=0,1$ :

Assumption A5. $\sup _{x \in \mathcal{S}(c)}\left|\hat{\pi}_{k} D_{j k}\left(x_{j}\right) /\left(\hat{\pi}_{0} D_{j 0}\left(x_{j}\right)+\hat{\pi}_{1} D_{j 1}\left(x_{j}\right)\right)\right|=O p\left(1+\sqrt{\frac{\log n}{n h^{3}}}\right)$.

We use A5 to give a mild bound simply to avoid the case where magnitude of both $D_{j k}\left(x_{j}\right), k=0,1$ are too large and close, but with opposite signs. A5 guarantees that the difference between the estimated marginal density $\hat{f}_{j k}\left(\hat{x}_{j}\right)$ and $f_{j k}\left(x_{j}\right)$ is able to be bounded by the same rate as when group eigenfunctions are equal. However, it is not a necessary condition for simply the asymptotic equivalence of $\log \hat{Q}_{J}^{*}(X)$ and $\log Q_{J}^{*}(X)$, and we can certainly relax its bound for Theorem 1 below.

Then, $\hat{f}_{j k}\left(\hat{x}_{j}\right)=\left(1 / \hat{\sigma}_{j k}\right) \hat{g}_{j k}\left(\hat{x}_{j}\right)$, we have Proposition 1 with proof in Supplementary:

Proposition 1. Under Assumptions A1- A5, when group eigenfunctions are unequal, the estimated marginal density $\hat{f}_{j k}$ using scores $\left\langle X_{i \cdot k}, \hat{\phi}_{j}\right\rangle$ achieves an asymptotic error bound: $\sup _{x \in \mathcal{S}(c)}\left|\hat{f}_{j k}\left(\hat{x}_{j}\right)-f_{j k}\left(x_{j}\right)\right|=O p\left\{h+\sqrt{\frac{\log n}{n h}}\right\}$, where the rate is the same as in Dai et al. (2017 [7]) where the group eigenfunctions are equal.

Assumption A6. Cumulative distribution functions (CDF) $F_{j k}$ of scores $X_{\cdot j k}$ are continuous and strictly increasing, with correspondent marginal densities $f_{j k}$ continuous and bounded. In addition, the $f_{j k}$ are bounded away from zero on any compact interval within their supports. 
A6 ensures that the scores $X_{. j k}$ as well as their monotonic transformations are atomless, and it also follows Condition 5 in Dai et al. (2017 [7]).

Then, in addition to the marginal densities, we establish the equivalence of $\Omega_{k}^{-1}$ and $\hat{\mathbf{\Omega}}_{k}^{-1}$ in $\log Q_{J}^{*}(X)$ and $\log \hat{Q}_{J}^{*}(X)$ respectively, as $n \rightarrow \infty$. As mentioned in Section 2.3, we calculate $\hat{\Omega}_{k}$ through rank correlations. Also, when $J$ is large, inverse of $\hat{\Omega}_{k}$ can be estimated by the graphical Dantzig selector (Yuan 2010 [35]), which solves the matrix inverse by connecting entries of the inverse correlation matrix to multivariate linear regression, and exploits the sparsity of the inverse matrices (Yuan 2010 [35]). Liu et al. (2012 [23]) provided a $q$-norm $O p$ bound of the difference between inverse Gaussian copula matrix and its estimation by the Dantzig estimator for high dimensional problems, and is extended here for the difference between $\Omega_{k}^{-1}$ and $\hat{\Omega}_{k}^{-1}$.

Our sparsity assumptions on the inverse correlation matrices follow the design of Yuan (2010 [35]) and Liu et al. (2012 [23]): let $\Omega_{k}$ belong to the class of matrices $\mathcal{C}(\kappa, \tau, M, J):=$ $\left\{\boldsymbol{\Omega}^{J \times J}: \boldsymbol{\Omega} \succ \mathbf{0}, \operatorname{diag}(\boldsymbol{\Omega})=\mathbf{1},\left\|\boldsymbol{\Omega}^{-1}\right\|_{1} \leq \kappa, \frac{1}{\tau} \leq \lambda_{\min }(\boldsymbol{\Omega}) \leq \lambda_{\max }(\boldsymbol{\Omega}) \leq \tau, \operatorname{deg}\left(\boldsymbol{\Omega}^{-1}\right) \leq M\right\}$, where $\kappa, \tau \geq 1$ are constants determining the tuning parameter in the graphical Dantzig selector, and the parameter $M$ bounding $\operatorname{deg}\left(\boldsymbol{\Omega}^{-1}\right)=\max _{1 \leq j \leq J} \sum_{j^{\prime}=1}^{J} I\left(\boldsymbol{\Omega}_{j j^{\prime}}^{-1} \neq 0\right)$ is dependent on $J$. Assuming these sparsity conditions, we have the following theorem:

Theorem 1. Under $A 1-A 6, \forall \epsilon>0$, as $n \rightarrow \infty$, there exists a sequence $J(n, \epsilon, M) \rightarrow \infty$, and a set $S$ dependent on $J(n, \epsilon, M), P(S) \geq 1-\epsilon$, such that

$$
P\left(S \cap\left\{\mathbb{1}\left\{\log \hat{Q}_{J}^{*}(X) \geq 0\right\} \neq \mathbb{1}\left\{\log Q_{J}^{*}(X) \geq 0\right\}\right\}\right) \rightarrow 0
$$

provided that $M J \sqrt{\log J}=o(\sqrt{n})$. 
Theorem 1 proves that under unequal group eigenfunctions, $\log \hat{Q}_{J}^{*}(X)$ using copulas retains the property in Theorem A1 of Dai et al. (2017 [7]) for the estimated Bayes classifiers with equal group eigenfunctions and independent scores: as $n \rightarrow \infty, \log \hat{Q}_{J}^{*}(X)$ gets arbitrarily close to the true Bayes classifier $\log Q_{J}^{*}(X)$, which enables us to discuss performance of our method using properties of the true Bayes classifier.

\subsection{Perfect classification when $X$ is a Gaussian process in both groups}

Let $X_{. . k}$ be a centered Gaussian process such that $X_{. . k}=\sum_{q=1}^{\infty} \sqrt{\lambda_{q k}} \xi_{q k} \phi_{q k}$, with $\xi_{q k} \sim$ $N(0,1)$, for $k=0,1$. We denote the $J \times J$ covariance matrix of scores $X_{\cdot j k}, 1 \leq j \leq J$, as $\mathbf{R}_{k}$, where its $\left(j, j^{\prime}\right)$-th entry equals $\operatorname{cov}\left(X_{\cdot j k}, X_{\cdot j^{\prime} k}\right)=\sum_{q=1}^{\infty} \lambda_{q k}\left\langle\phi_{q k}, \phi_{j}\right\rangle\left\langle\phi_{q k}, \phi_{j^{\prime}}\right\rangle$, and its eigenvalues are $d_{1 k}, \ldots, d_{J k}$. Let $\vec{\mu}_{J}$ be a length- $J$ vector $\left(\mu_{1}, \ldots, \mu_{J}\right)^{T}$ by projecting $\mu_{d}$ on first $J$ basis, $\mu_{j}=\left\langle\mu_{d}, \phi_{j}\right\rangle$. By the law of total covariance and the result that the trace of a matrix equals the sum of its eigenvalues, we derive the following relationship between the two sets of eigenvalues (i.e. $\lambda_{j}, \lambda_{j k}$ and $d_{j k}$ ): $\sum_{j=1}^{J} \lambda_{j}=\pi_{1} \sum_{j=1}^{J} d_{j 1}+\pi_{0} \sum_{j=1}^{J} d_{j 0}+\pi_{1} \pi_{0} \sum_{j=1}^{J} \mu_{j}^{2}$, and $\sum_{j=1}^{J} d_{j k}=\sum_{j=1}^{J} \sum_{q=1}^{\infty} \lambda_{q k}\left\langle\phi_{q k}, \phi_{j}\right\rangle^{2}$. The following assumption is standard in functional data for the distribution of $X$, and ensures that $d_{j k}>0,1 \leq j \leq J, k=0,1$ :

Assumption A7. Both the group covariance operators, $G_{1}, G_{0}$, and the covariance matrices $\mathbf{R}_{0}, \mathbf{R}_{1}$ are bounded and positive definite, and $\mu_{d} \in \mathcal{L}^{2}(\mathcal{T})$.

When $X$ is Gaussian in both groups, $\log Q_{J}^{*}(X)$ is a quadratic form in $\mathbf{X}_{J}\left(\mathbf{X}_{J}\right.$ is a length $J$ vector with $j$-th entry $\left.\left\langle X, \phi_{j}\right\rangle\right)$ :

$$
\log Q_{J}^{*}(X)=-\frac{1}{2}\left(\mathbf{X}_{J}-\vec{\mu}_{J}\right)^{T} \mathbf{R}_{1}^{-1}\left(\mathbf{X}_{J}-\vec{\mu}_{J}\right)+\frac{1}{2} \mathbf{X}_{J}^{T} \mathbf{R}_{0}^{-1} \mathbf{X}_{J}+\log \sqrt{\frac{\left|\mathbf{R}_{0}\right|}{\left|\mathbf{R}_{1}\right|}}
$$

With potentially unequal group eigenfunctions, entries in $\mathbf{X}_{J}$ at $Y=k$ can be correlated, 
which complicates the distribution of $\log Q_{J}^{*}(X)$ at each group.

Therefore, we implement a linear transformation of $\mathbf{X}_{J}$ in Steps i) - iii):

i) The eigendecomposition of the matrix product gives $\mathbf{R}_{0}^{1 / 2} \mathbf{R}_{1}^{-1} \mathbf{R}_{0}^{1 / 2}=\mathbf{P}^{T} \boldsymbol{\Delta} \mathbf{P}$, where $\boldsymbol{\Delta}=\operatorname{diag}\left\{\Delta_{1}, \ldots, \Delta_{J}\right\}, \Delta_{j}$ as eigenvalues of $\mathbf{R}_{0}^{1 / 2} \mathbf{R}_{1}^{-1} \mathbf{R}_{0}^{1 / 2}$. By the equivalence of determinants, $\prod_{j=1}^{J} \frac{d_{j 0}}{d_{j 1}}=\prod_{j=1}^{J} \Delta_{j}$. Also, $\Delta_{j}>0$ for all $j$ under A7;

ii) Let $\mathbf{Z}=\mathbf{R}_{0}^{-1 / 2} \mathbf{X}_{J}, \mathbf{U}=\mathbf{P Z}$;

iii) When $k=0$, the $j$-th entry $U_{j}$ of vector $\mathbf{U}$ has a standard Gaussian distribution; at $k=1, U_{j} \sim N\left(-b_{j}, 1 / \Delta_{j}\right)$, with $b_{j}$ the $j$-th entry of $\mathbf{b}=-\mathbf{P R}_{0}^{-1 / 2} \vec{\mu}_{J}$.

Consequently, entries of $\mathbf{U}$ are uncorrelated for both $k=0$ and 1, and Eq.(5.1) becomes

$$
\log Q_{J}^{*}(X)=-\frac{1}{2} \sum_{j=1}^{J} \Delta_{j}\left(U_{j}+b_{j}\right)^{2}+\frac{1}{2} \sum_{j=1}^{J} U_{j}^{2}+\frac{1}{2} \sum_{j=1}^{J} \log \Delta_{j}
$$

and the asymptotic behaviors of the Bayes classifier for Gaussian processes are concluded:

Theorem 2. With A7, when random function $X$ is a Gaussian process at both $Y=0$ and 1 , and group eigenfunctions of $G_{0}, G_{1}$ are unequal, functional Bayes classifier $\mathbb{1}\left\{\log Q_{J}^{*}(X)>\right.$ $0\}$ achieves perfect classification when either $\left\|\mathbf{R}_{0}^{-1 / 2} \vec{\mu}_{J}\right\|^{2} \rightarrow \infty$, or $\sum_{j=1}^{J}\left(\Delta_{j}-1\right)^{2} \rightarrow \infty$, as $J \rightarrow \infty$. Otherwise its error rate $\operatorname{err}\left(\mathbb{1}\left\{\log Q_{J}^{*}(X)>0\right\}\right) \nrightarrow 0$.

Theorem 2 is a natural extension of Theorem 2 in Dai et al. (2017 [7]). It again reveals that the error rate of the Bayes classifier approaches zero asymptotically when $\Pi_{1}$ and $\Pi_{0}$ are sufficiently different in either the group means or the scores' variances. In addition, recognizing the different correlation patterns between group scores is also helpful for 
improving classification accuracy. Instead of adopting $\mu_{j} / \sqrt{\lambda_{j 0}}$ and $\lambda_{j 0} / \lambda_{j 1}$ to build conditions for perfect classification as in Dai et al. (2017 [7]), we use the transformed $\mathbf{R}_{0}^{-1 / 2} \vec{\mu}_{J}$ and $\Delta_{j}$ to accommodate the potentially unequal group eigenfunctions as well as dependent scores. For the special case when eigenfunctions are actually equal, the covariance matrices $\mathbf{R}_{k}=\operatorname{diag}\left\{\lambda_{1 k}, \ldots, \lambda_{J k}\right\}$ with $\Delta_{j}=\lambda_{j 0} / \lambda_{j 1}$, and consequently the two conditions in Theorem 2 become the same as the ones proposed in Dai et al. (2017 [7]). The proof of Theorem 2 is in Section S6.2 of the Supplementary Materials.

\subsection{When $X$ is non-Gaussian process}

For non-Gaussian processes, when the projected scores $X_{\cdot j k}$ for $1 \leq j \leq J$ fit a Gaussian copula model, i.e., they are meta-Gaussian distributed, we derive sufficient conditions in terms of marginal densities $f_{j k}$ and score correlations, in order to achieve an asymptotically zero misclassification rate.

First, we let $\mathbf{u}_{k}=\left(u_{1 k}, \ldots, u_{J k}\right)^{T}$ be a length $J$ random vector with $u_{j k}=\Phi^{-1}\left(F_{j k}\left(X_{\cdot j .}\right)\right)$, where $\Phi(\cdot)$ is the CDF of $N(0,1)$. When $Y=k,\left(u_{j k} \mid Y=k\right) \sim N(0,1)$, and $\operatorname{var}\left(\mathbf{u}_{k} \mid Y=k\right)=$ $\Omega_{k}$ as denoted before. Let the eigendecomposition be $\boldsymbol{\Omega}_{k}=\mathbf{V}_{k} \mathbf{D}_{k} \mathbf{V}_{k}^{T}$, with $\mathbf{D}_{k}$ the diagonal matrix with eigenvalues $\omega_{j k}, j=1, \ldots, J$. On the other hand, $u_{j k} \mid Y=k^{\prime}$ follows a more complicated distribution when $k^{\prime} \neq k$. We denote $\operatorname{var}\left(\mathbf{u}_{\mathbf{k}} \mid Y=k^{\prime}\right)=\mathbf{M}_{k}$ with eigendecomposition $\mathbf{M}_{k}=\mathbf{U}_{k} \tilde{\mathbf{D}}_{k} \mathbf{U}_{k}^{T}$, and the eigenvalues of $\mathbf{M}_{k}$ are $v_{j k}, j=1, \ldots, J$.

Therefore the $\log$ density ratio $\log Q_{J}^{*}(X)$ in the Bayes classifier with Gaussian copula 
can be represented as

$$
\begin{aligned}
\log Q_{J}^{*}(X) & =\sum_{j=1}^{J} \log \frac{f_{j 1}\left(X_{\cdot j .}\right)}{f_{j 0}\left(X_{\cdot j .}\right)}+\frac{1}{2} \log \frac{\left|\boldsymbol{\Omega}_{0}\right|}{\left|\boldsymbol{\Omega}_{1}\right|}-\frac{1}{2} \mathbf{u}_{1}^{T}\left(\boldsymbol{\Omega}_{1}^{-1}-\mathbf{I}\right) \mathbf{u}_{1}+\frac{1}{2} \mathbf{u}_{0}^{T}\left(\boldsymbol{\Omega}_{0}^{-1}-\mathbf{I}\right) \mathbf{u}_{0} \\
& =\sum_{j=1}^{J} \log \frac{f_{j 1}\left(X_{\cdot j .}\right)}{f_{j 0}\left(X_{\cdot j \cdot}\right)} / \frac{\sqrt{\omega_{j 1}}}{\sqrt{\omega_{j 0}}}-\frac{1}{2} \mathbf{u}_{1}^{T}\left(\boldsymbol{\Omega}_{1}^{-1}-\mathbf{I}\right) \mathbf{u}_{1}+\frac{1}{2} \mathbf{u}_{0}^{T}\left(\boldsymbol{\Omega}_{0}^{-1}-\mathbf{I}\right) \mathbf{u}_{0} .
\end{aligned}
$$

Similar to A7, we make an assumption on the covariances of $\mathbf{u}_{k}$ conditional on $Y$ :

Assumption A8. Matrices $\boldsymbol{\Omega}_{k}$ and $\mathbf{M}_{k}, k=0,1$, are bounded and positive definite.

Next, we define a sequence of ratios $g_{j}, j=1,2, \ldots$, by $g_{j}=\frac{f_{j 1}\left(X_{. j .}\right)}{f_{j 0}\left(X_{. j .}\right)} / \frac{\sqrt{\omega_{j 1}}}{\sqrt{\omega_{j 0}}}$, where $g_{j}$ compares the ratio of the marginal densities to the ratio of the eigenvalues of the correlation matrices. In addition, let

$$
s_{j k}=\frac{\operatorname{var}\left(\left\langle V_{j k}, \mathbf{u}_{k}\right\rangle \mid Y=k\right)}{\operatorname{var}\left(\left\langle V_{j k}, \mathbf{u}_{k}\right\rangle \mid Y=k^{\prime}\right)}=\frac{\mathbf{V}_{j k}^{T} \mathbf{\Omega}_{k} \mathbf{V}_{j k}}{\mathbf{V}_{j k}^{T} \mathbf{M}_{k} \mathbf{V}_{j k}}=\frac{\omega_{j k}}{\sum_{q=1}^{J} C_{(j, q) k}^{2} v_{q k}},
$$

where $C_{(j, q) k}=\left\langle\mathbf{U}_{q k}, \mathbf{V}_{j k}\right\rangle, \sum_{q=1}^{J} C_{(j, q) k}=1$, and $\mathbf{U}_{q k}, \mathbf{V}_{j k}$ are respectively $q$-th and $j$-th columns of eigenvector matrices $\mathbf{U}_{k}, \mathbf{V}_{k}$. As a consequence, $s_{j k}$ compares the $j$-th eigenvalue of $\boldsymbol{\Omega}_{k}$ against a convex combination of $\mathbf{M}_{k}$ 's eigenvalues, whose individual weights are determined by projecting $\mathbf{V}_{j k}$ on $\mathbf{M}_{k}$ 's eigenvectors $\mathbf{U}_{q k}$.

In terms of the sequences $g_{j}$ and $s_{j k}$, for $j=1,2, \ldots$, we derive the following theorem for non-Gaussian processes, whose proof is in Section S6.3 of the Supplementary Materials.

Theorem 3. With assumptions A6, A7 and A8, when the projected scores $X_{\cdot j k}, j=1, \ldots, J$, are meta-Gaussian distributed at each group $\Pi_{k}$, perfect classification by the Bayes classifier $\mathbb{1}\left\{\log Q_{J}^{*}(X)>0\right\}$ is achieved asymptotically, if a subsequence $g_{r}^{*}=g_{j_{r}}$ of $g_{j}$ exists, with corresponding $s_{j_{r} k}$, such that one of the following conditions is satisfied as $r \rightarrow \infty$ : 
a) $g_{j_{r}}=o p(1)$, and $s_{j_{r} 0} \rightarrow 0$;

b) $1 / g_{j_{r}}=o p(1)$, and $s_{j_{r} 1} \rightarrow 0$;

or when $g_{j_{r}}$ has distinct behaviors in subgroups:

c) $g_{j_{r}}=o p(1)$ at $Y=1,1 / g_{j_{r}}=o p(1)$ at $Y=0$, with both $s_{j_{r} 0}$ and $s_{j_{r} 1} \rightarrow 0$;

d) $1 / g_{j_{r}}=o p(1)$ at $Y=1$, and $g_{j_{r}}=o p(1)$ at $Y=0$.

Based on the structure of the log density ratio as described in Eq.(5.2), Theorem 3 discusses the occurrence of perfect classification in two aspects: $g_{j}$ which mainly depicts the relative magnitude of score marginal densities at each $k=0,1$; and also $s_{j k}$ which compares the correlation between scores conditioned at each group. Either part showing enough disparity between groups results in perfect classification.

For example, in Theorem 3 a), when there exists a subsequence $g_{j_{r}} \rightarrow 0$ in probability, indicating the dominance of marginal densities by group $Y=0$, the misclassification tends to occur at $Y=1$. However, as $s_{j_{r} 0} \rightarrow 0$, covariance of $\mathbf{u}_{0}$ conditioned at $Y=1$ would be much larger than at $Y=0$. As a consequence, the nonnegative $\mathbf{u}_{0}^{T} \boldsymbol{\Omega}_{0}^{-1} \mathbf{u}_{0}^{T}$ in Eq.(5.2) with large variation when $Y=1$ would compensate to eventually avoid misclassifying $X$ to group 0 . When $g_{j_{r}}$ behaves perfectly as in case d), where the correspondent group marginal densities are dominant in each subgroup $Y=k$, we do not need to impose requirements on the copula correlation to achieve perfect classification.

Remark. Theorem 3 provides sufficient yet not necessary conditions for the Bayes classifier to achieve asymptotic perfect classification under unequal group eigenfunctions. Due to the optimality of the Bayes classifier in minimizing zero-one loss, various conditions from other functional classifiers to achieve asymptotically zero error also work here. For example, 
Delaigle and Hall (2012a [9]) proposed conditions in terms of group eigenvalues and mean difference for the functional centroid classifier to reach perfect classification. These also work as sufficient conditions for $\mathbb{1}\left\{\log Q_{J}^{*}(X)>0\right\}$ in our case. With a copula model, which is not found in previous work, Theorem 3 utilizes the relation between scores' marginal densities and correlations to reduce the error rate to zero asymptotically.

\section{Discussion}

\subsection{Remarks}

Our copula-based Bayes classifiers remove the assumptions of equal group eigenfunctions and independent scores. As our two examples show, it is not uncommon to have unequal group eigenfunctions (see Fig. S4 and Fig. S8). The new methods also prove to have stronger performance in dimension reduction than the original BC. Simulation results prove the strength of our method in distinguishing groups by differences in their functional means as well as their covariance functions. We examined the two choices of projection directions, PC and PLS. PLS can detect location differences on eigenfunctions corresponding to smaller eigenvalues. We discussed new conditions for the estimated classifier to be asymptotically equivalent to the true Bayes classifier and for the perfect classification to occur, which differed from previous work due to the unequal group eigenfunction setting. We also imposed sparsity conditions on the inverse of copula correlations.

\subsection{Future Work}

In the future work, we would like to further the study of copula-based classification to the problem with multiple functional covariates. Some previous works discussed this situation in the framework of functional generalized models: Crainiceanu et al. (2009) [5] proposed 
a generalized multilevel regression model where there are repeated curve measurements for each subject; Zhu et al. (2010) [36] discussed an FGLM approach for classification of multilevel functions with Bayesian variable selection; Li et al. (2012) [22] present a generalized functional linear model where there are both functional and multivariate covariates, and used a semiparametric single-index function to model the interaction between them. We plan to approach the problem from a different angle, using functional Bayes classification again due to its strong performance in the single functional predictor case. Also, as it's natural to assume that the response depends on the covariates and their interactions, it becomes more important for our method to model the dependency between projected scores. Another aspect we would like to consider is about how to choose a proper functional basis for multiple functional predictors.

\section{Supplementary Materials}

The Supplementary Materials for this document contain additional results for the simulations, for the fractional anisotropy (FA) example, and for the example using truck emissions. They also contain proofs of Theorems 1, 2, and 3.

\section{Acknowledgements}

The MRI/DTI data in the refund package were collected at Johns Hopkins University and the Kennedy-Krieger Institute.

\section{References}

[1] Benko, M., Härdle, W., and Kneip, A. (2009). Common functional principal components. The Annals of Statistics, $37(1): 1-34$.

[2] Chen, X. and Fan, Y. (2006). Estimation of copula-based semiparametric time series models. Journal of Econo- 
metrics, 130(2):307-335.

[3] Cholaquidis, A., Fraiman, R., Kalemkerian, J., and Llop, P. (2016). A nonlinear aggregation type classifier. Journal of Multivariate Analysis, 146:269-281.

[4] Clark, N. N., Gautam, M., Wayne, W. S., Lyons, D. W., Thompson, G., and Zielinska, B. (2007). Heavy-duty vehicle chassis dynamometer testing for emissions inventory, air quality modeling, source apportionment and air toxics emissions inventory. Coordinating Research Council, incorporated.

[5] Crainiceanu, C. M., Staicu, A.-M., and Di, C.-Z. (2009). Generalized multilevel functional regression. Journal of the American Statistical Association, 104(488):1550-1561.

[6] Cuevas, A., Febrero, M., and Fraiman, R. (2007). Robust estimation and classification for functional data via projection-based depth notions. Computational Statistics, 22(3):481-496.

[7] Dai, X., Müller, H.-G., and Yao, F. (2017). Optimal bayes classifiers for functional data and density ratios. Biometrika, 104(3):545-560.

[8] Delaigle, A. and Hall, P. (2010). Defining probability density for a distribution of random functions. The Annals of Statistics, 38(2):1171-1193.

[9] Delaigle, A. and Hall, P. (2012a). Achieving near perfect classification for functional data. Journal of the Royal Statistical Society: Series B (Statistical Methodology), 74(2):267-286.

[10] Escoufier, Y. (1970). Echantillonnage dans une population de variables aléatoires réelles. Department de math.; Univ. des sciences et techniques du Languedoc.

[11] Genest, C., Ghoudi, K., and Rivest, L.-P. (1995). A semiparametric estimation procedure of dependence parameters in multivariate families of distributions. Biometrika, 82(3):543-552.

[12] Gijbels, I., Omelka, M., and Veraverbeke, N. (2012). Multivariate and functional covariates and conditional copulas. Electronic Journal of Statistics, 6:1273-1306.

[13] Goldsmith, J., Crainiceanu, C. M., Caffo, B., and Reich, D. (2012). Longitudinal penalized functional regression 
for cognitive outcomes on neuronal tract measurements. Journal of the Royal Statistical Society: Series C (Applied Statistics), 61(3):453-469.

[14] Goldsmith, J., Scheipl, F., Huang, L., Wrobel, J., Gellar, J., Harezlak, J., McLean, M., Swihart, B., Xiao, L., Crainiceanu, C., Reiss, P., Chen, Y., Greven, S., Huo, L., Kundu, M., Park, S., Miller, D. s., and Staicu, A.-M. (2018). refund: Regression with functional data. $R$ package version, 0.1(17).

[15] Hofert, M., Kojadinovic, I., Maechler, M., and Yan, J. (2018). copula: Multivariate Dependence with Copulas. R package version 0.999-19.1.

[16] James, G. M. (2002). Generalized linear models with functional predictors. Journal of the Royal Statistical Society: Series B (Statistical Methodology), 64(3):411-432.

[17] James, G. M. and Hastie, T. J. (2001). Functional linear discriminant analysis for irregularly sampled curves. Journal of the Royal Statistical Society: Series B (Statistical Methodology), 63(3):533-550.

[18] Kauermann, G., Schellhase, C., and Ruppert, D. (2013). Flexible copula density estimation with penalized hierarchical b-splines. Scandinavian Journal of Statistics, 40(4):685-705.

[19] Kendall, M. G. (1948). Rank correlation methods.

[20] Kruskal, W. H. (1958). Ordinal measures of association. Journal of the American Statistical Association, $53(284): 814-861$.

[21] Li, B. and Yu, Q. (2008). Classification of functional data: A segmentation approach. Computational Statistics E Data Analysis, 52(10):4790-4800.

[22] Li, Y., Wang, N., and Carroll, R. J. (2010). Generalized functional linear models with semiparametric singleindex interactions. Journal of the American Statistical Association, 105(490):621-633.

[23] Liu, H., Han, F., Yuan, M., Lafferty, J., and Wasserman, L. (2012). High-dimensional semiparametric gaussian copula graphical models. The Annals of Statistics, 40(4):2293-2326.

[24] Mashal, R. and Zeevi, A. (2002). Beyond correlation: Extreme co-movements between financial assets. Unpub- 
lished, Columbia University.

[25] McLean, M. W., Hooker, G., and Ruppert, D. (2015). Restricted likelihood ratio tests for linearity in scalar-onfunction regression. Statistics and Computing, 25(5):997-1008.

[26] McLean, M. W., Hooker, G., Staicu, A.-M., Scheipl, F., and Ruppert, D. (2014). Functional generalized additive models. Journal of Computational and Graphical Statistics, 23(1):249-269.

[27] Mevik, B.-H., Wehrens, R., and Liland, K. H. (2011). pls: Partial least squares and principal component regression. $R$ package version, $2(3)$.

[28] Müller, H.-G., Stadtmüller, U., et al. (2005). Generalized functional linear models. Annals of Statistics, $33(2): 774-805$.

[29] Preda, C., Saporta, G., and Lévéder, C. (2007). Pls classification of functional data. Computational Statistics, $22(2): 223-235$.

[30] Ramsay, J. O. and Silverman, B. W. (2005). Functional data analysis. New York: Springer.

[31] Rossi, F. and Villa, N. (2006). Support vector machine for functional data classification. Neurocomputing, $69(7-9): 730-742$.

[32] Ruppert, D. and Matteson, D. S. (2015). Statistics and Data Analysis for Financial Engineering with $R$ examples. Springer.

[33] Shang, Z., Cheng, G., et al. (2015). Nonparametric inference in generalized functional linear models. The Annals of Statistics, 43(4):1742-1773.

[34] Sheather, S. J. and Jones, M. C. (1991). A reliable data-based bandwidth selection method for kernel density estimation. Journal of the Royal Statistical Society: Series B (Methodological), 53(3):683-690.

[35] Yuan, M. (2010). High dimensional inverse covariance matrix estimation via linear programming. Journal of Machine Learning Research, 11(Aug):2261-2286. 
[36] Zhu, H., Vannucci, M., and Cox, D. D. (2010). A bayesian hierarchical model for classification with selection of functional predictors. Biometrics, 66(2):463-473.

Department of Statistics and Data Science, Cornell University

E-mail: wh365@cornell.edu

School of Operations Research and Information Engineering, and Department of Statistics and Data Science, Cornell University

E-mail: dr24@cornell.edu 